\title{
Pattern avoidance in permutations: linear and cyclic orders
}

\author{
Antoine Vella* \\ Dept. of Combinatorics and Optimization, University of Waterloo \\ 200 University Avenue West, N2L 3G1 Waterloo, Canada \\ avella@math.uwaterloo.ca
}

Submitted: Jun 10, 2003; Accepted: Oct 28, 2003; Published: Nov 7, 2003

MR Subject Classifications: 05C88, 05C89

\begin{abstract}
We generalize the notion of pattern avoidance to arbitrary functions on ordered sets, and consider specifically three scenarios for permutations: linear, cyclic and hybrid, the first one corresponding to classical permutation avoidance. The cyclic modification allows for circular shifts in the entries. Using two bijections, both ascribable to both Deutsch and Krattenthaler independently, we single out two geometrically significant classes of Dyck paths that correspond to two instances of simultaneous avoidance in the purely linear case, and to two distinct patterns in the hybrid case: non-decreasing Dyck paths (first considered by Barcucci et al.), and Dyck paths with at most one long vertical or horizontal edge. We derive a generating function counting Dyck paths by their number of low and high peaks, long horizontal and vertical edges, and what we call sinking steps. This translates into the joint distribution of fixed points, excedances, deficiencies, descents and inverse descents over 321-avoiding permutations. In particular we give an explicit formula for the number of 321-avoiding permutations with precisely $k$ descents, a problem recently brought up by Reifegerste. In both the hybrid and purely cyclic scenarios, we deal with the avoidance enumeration problem for all patterns of length up to 4. Simple Dyck paths also have a connection to the purely cyclic case; here the orbit-counting lemma gives a formula involving the Euler totient function and leads us to consider an interesting subgroup of the symmetric group.
\end{abstract}

\section{Introduction}

Pattern avoidance in permutations has received much attention in the last few years. The basic idea is the following: if we write a permutation as a sequence of integers $a_{1} a_{2}, \ldots a_{n}$, then we can consider subsequences to be "occurrences" of smaller permutations by keeping track of the order in which the chosen entries appear, and their values. So for example 523 would be an occurrence of 312 in 652431 . Often the term "permutation" is used to mean a bijective mapping of an arbitrary (typically finite) set into itself; however, any

* Research financed by the EC's IHRP Programme, within the Research Training Network "Algebraic Combinatorics in Europe", grant HPRN-CT-2001-00272, while the author was at Chalmers Tekniska Högskola, Göteborg, Sweden. 
formalization of the concept of avoidance in the usual sense requires the set to be equipped with a linear (total) order. Once we have such a formalization, we can consider situations in which the order is not necessarily linear. Here we propose to take what appears to be a natural next step: go from linear to cyclic.

In [8], in order to obtain a combinatorialist's generalization of the concept of a permutation from the finite to the infinite, Cameron regards a permutation as a pair of total orders on the ground set. In this context, he also considers subpermutations, cyclic orders and circular permutations. His definition naturally extends to an arbitrary number of orders; the one we shall give generalizes in a different direction. For the specific cases we shall consider in this paper, our definitions are essentially equivalent to Cameron's, and can be simplified without loss of rigour; however, we wish to emphasize that they generalize the concept of pattern avoidance to arbitrary functions whose domain and codomain are ordered sets, and open up a myriad questions in this regard.

Here by ordered set we mean a set $X$ equipped with an arbitrary " $k$-ary relation", that is a subset $\mathcal{T}_{X}$ of the cartesian product $X^{k}$, for some positive integer $k$. Two standard examples are the familiar linear (total) orders, obtained by taking a binary relation satisfying the properties of antisymmetry, transitivity, reflexivity and decisiveness ${ }^{1}$, and cyclic orders, given by a ternary relation satisfying certain properties which we shall specify in Section 1.2. In both cases, we have an essentially (up to isomorphism) unique way of constructing an order of the prescribed type on a given set. As prototypes of finite linear and cyclically ordered sets, we may take $X$ to be simply the set $I_{n}$ of the first $n$ positive integers, with the binary relation consisting of all pairs $(i, j)$ with $i \leq j$ for the linear order, while a cyclic order is given by all triples $(i, j, k),(j, k, i),(k, i, j)$ with $i \leq j \leq k$.

A subset $Y$ of $X$ inherits an ordered structure given by the subset of $X^{k}\left\{t \in \mathcal{T}_{X} \mid t_{i} \in\right.$ $Y \forall i\}$, where $t_{i}$ denotes the $i$-th coordinate of $t$; that is, we take all tuples whose coordinates all take values in $Y$. In the above examples, the inherited order turns out to be essentially the same as the one we would construct directly on $Y$ itself. An orderisomorphism of two ordered sets $X, Y$ is a bijection $\sigma$ such that, for all $k$-tuples $t \in X^{k}$, we have $t \in \mathcal{T}_{X}$ if and only if the corresponding tuple $\left(\sigma\left(t_{1}\right), \sigma\left(t_{2}\right), \ldots, \sigma\left(t_{s}\right)\right)$ belongs to $\mathcal{T}_{Y}$. Given any two linearly ordered sets, there is a unique isomorphism between them if and only if they have the same cardinality, and none otherwise; if instead we have two finite cyclically ordered sets of cardinalities $n_{1}, n_{2}$, then again there exist isomorphisms if and only if $n_{1}=n_{2}(=n)$, and in this case there are precisely $n$ of them. For example, if we write the letters of the English alphabet in clockwise order on a circle, and take the cyclic order given by all triples which can be read off the circle in clockwise fashion, then one order isomorphism of $I_{26}$ with the cyclic order onto the English alphabet is the map $1 \mapsto e, 2 \mapsto f, \ldots, 22 \mapsto z, 23 \mapsto a, \ldots, 26 \mapsto d$, and all others are "rotations" of this.

Given functions $\gamma: A \rightarrow B$ and $\delta: B \rightarrow C, \gamma \circ \delta$ denotes the function $a \mapsto \delta(\gamma(a))$ (note this notation may be in conflict with that used by several authors). An order function is a function whose domain and codomain are both ordered sets. Given order functions $f: D \rightarrow E$ and $g: F \rightarrow G$, we say that $f$ and $g$ are order-equivalent if there exist order-isomorphisms $\alpha: D \rightarrow F$ and $\beta: g(F) \rightarrow f(D)$ such that $f=\alpha \circ g \circ \beta$, where

\footnotetext{
${ }^{1}$ This is the requirement that any two elements be comparable.
} 
$g(F)$ and $f(D)$ inherit their orders from $G$ and $E$ respectively. If $h$ is an order function, an occurrence of $h$ is a subset $S$ of the domain of $f$ such that $\left.f\right|_{S}$ is order-equivalent to $h$.

Consider for example the linearly ordered sets $I_{5}$ and $I_{8}$, the set $\Sigma$ of letters of the English alphabet, with the cyclic order defined above, and the order functions $\chi: I_{8} \rightarrow \Sigma$ and $\psi: I_{5} \rightarrow \Sigma$

$$
\chi: \begin{array}{llllllll}
1 & 2 & 3 & 4 & 5 & 6 & 7 & 8 \\
p & a & t & t & e & r & n & s
\end{array} \quad \psi: \begin{array}{llllll}
1 & 2 & 3 & 4 & 5 \\
a & c & c & d & b
\end{array}
$$

Then the set $\{1,3,4,7,8\}$ inherits a linear order from $I_{8}$, the sets $\{a, b, c, d\}$ and $\{n, p, s, t\}$ inherit cyclic orders from $\Sigma$ and the order isomorphisms

\begin{tabular}{lllll||llll}
1 & 2 & 3 & 4 & 5 & $a$ & $b$ & $c$ & $d$ \\
1 & 3 & 4 & 7 & 8 & $p$ & $s$ & $t$ & $n$
\end{tabular}

show that the function

$\begin{array}{lllll}1 & 3 & 4 & 7 & 8 \\ p & t & t & n & s\end{array}$

is order-equivalent to $\psi$, and therefore the set $\{1,3,4,7,8\} \subseteq I_{8}$ is an occurrence of $\psi$ in $\chi$.

If no subset of the domain of $f$ is an occurrence of $h$, then $f$ avoids $h$. Equivalently, $f$ is $h$-avoiding. This also extends to simultaneous avoidance, i.e. if $Z$ is a set of order functions, $f$ avoids $Z$ (or is $Z$-avoiding) if it avoids all elements of $Z$. Also, an occurrence of $Z$ is an occurrence of an element of $Z$. It is easy to check that order-isomorphism is an equivalence relation, and that avoidance is independent of the particular representative of the equivalence class. More precisely, if $h_{1}, h_{2}$ are order-isomorphic order functions, then $S$ is an occurrence of $h_{1}$ if and only if it is an occurrence of $h_{2}$, and if $f, g$ are order-isomorphic as in the definition above, then $S$ is an occurrence of $h$ in $f$ if and only if $\alpha(S)$ is an occurrence of $h$ in $g$.

Thus it makes sense to speak of one equivalence class avoiding another, and a pattern could be defined as an equivalence class of order functions (which might as well be surjective). In keeping with current terminology, we shall reserve the term "pattern" for the equivalence classes being avoided.

Graphs provide other examples of pattern avoidance in the above sense; if for example we take the order on the domain to be an arbitrary symmetric reflexive binary relation, and the codomain to be the linearly ordered set $I_{s}$, then we are dealing with $s$-coloured graphs avoiding a subgraph with a prescribed $t$-labelling $\left(I_{t}\right.$ being the codomain of the pattern), in the sense that the labels of a copy of the subgraph in the graph may not have the same relative order as those on the subgraph (via any graph-isomorphism). If we take the pattern to be just an edge labelled with a constant, then we are dealing with properly $n$-coloured graphs, and for a fixed graph the problem of enumerating the order functions avoiding this pattern is "solved" by the chromatic polynomial.

Different interesting enumeration problems arise in different contexts; for example, we could take the order functions to be the identity mappings from graphs to themselves, in 
which case we are dealing with graphs avoiding a fixed subgraph. An asymptotic version of this problem (which also fits into the context of Cameron) has been solved in terms of threshold functions; see for example [2], Chapter 4.

However, in this paper we shall not venture far from classical permutation avoidance; we shall consider only bijective functions, in the following scenarios:

1. linear orders on the domain and the codomain - this gives classical permutation avoidance;

2. a cyclic order on the domain and a linear order on the codomain - in this case, taking order-equivalent functions corresponds to "wrapping around" in the domain, and we shall call the equivalence classes cyclic arrangements; e.g. 35412, 54123, 41235, 12354 and $23541^{2}$ all correspond to the same cyclic arrangement;

3. cyclic orders on both the domain and codomain - in this case, taking order-equivalent functions corresponds to "wrapping around" independently both in the domain and in the codomain (not necessarily by the same "shift"), and we shall use the term orbits for the equivalence classes; e.g. 35412,54123 and $32451^{2}$.

The case of a linear order on the domain and a cyclic order on the codomain is entirely analogous to the the second one above. Note that, in the literature, the term circular permutations is variously used to refer to the equivalence classes in one or the other of the last two cases.

In scenarios (2) and (3) above, although the problem of finding the equivalence classes avoiding a given pattern (equivalence class) is reducible to that of determining the set $A$ of permutations avoiding a certain set $Z$ of patterns, our techniques for determining $A$ make use of the cyclic structure and do not extend to an arbitrary set of patterns of the same length; moreover, in scenario (3) taking equivalence classes on $A$ is non-trivial and therefore the enumeration problem becomes more complicated.

We remark here that the orders we are considering have the following very important properties:

- they are parametrizable with cardinality, i.e. given a finite set, we can construct the corresponding order in a unique (up to order-isomorphism) way, and the result depends only on the cardinality of the given set;

- the inherited order depends only on the cardinality of the subsets, i.e. for a fixed integer $k$, any two subsets of cardinality $k$ with the inherited order structure are order-isomorphic;

- inheritance is well-behaved, in the sense that the inherited order on a subset $S$ agrees with the one constructed a priori on $S$.

\footnotetext{
${ }^{2}$ If necessary, refer to Section 1.2 for an explanation of this notation.
} 
Thus in our context it is sufficient to specify the cardinalities of the domain and codomain in question, and since we shall deal exclusively with bijections, we might as well assume them to be the same set. Clearly, if this set has cardinality $n$, we may take it to be $I_{n}$, as long as we do not feel necessarily bound to the usual order on the integers. Since modular arithmetic offers a convenient way of dealing with cyclic orders on $I_{n}$ (except for letting $n$ replace the usual 0 ), we shall always indeed assume that our functions are permutations from $I_{n}$ onto itself.

\subsection{Overview}

In Section 2 we deal with classical permutation avoidance, with reference to two different bijections, both discovered independently by Krattenthaler [15] and Deutsch, that relate permutation avoidance to Dyck paths. We single out two geometrically significant classes of Dyck paths which, under these bijections, correspond to $\{132,3241\}$-avoiding permutations and $\{321,2143\}$-avoiding permutations respectively, namely non-decreasing Dyck paths, first considered by Barcucci et al. [3], and what we call simple Dyck paths. Simple Dyck paths are characterized by the property of having at most one long vertical edge or at most one long horizontal edge, where we consider an edge to be "long" if it consists of at least two consecutive steps (of the same kind). These classes of Dyck paths enable us to give new proofs of results needed in Sections 3 and 4, first obtained by Billey et al. [5] and West [29]. In doing so, we give a bijective construction of non-decreasing Dyck paths (the zigzag construction), use it to refine the enumeration of these paths of Barcucci et al. in terms of the number of valleys, translate this into a simple explicit formula in $n$ and $k$ for the number of $\{132,3241\}$-avoiding permutations of length $n$ with precisely $k$ descents and characterize $\{321,2143\}$-avoiding permutations in terms of Grassmannian permutations. We also derive a generating function counting Dyck paths simultaneously by the number of hilltops and mountain-tops (peaks at height one or more respectively), long horizontal and vertical edges and sinking steps - horizontal steps which are not the first step of the edge they belong to. These statistics on Dyck paths translate into statistics on 321-avoiding permutations, namely fixed points, excedances, descents, dips (descents in the inverse permutation, also called "inverse descents"), and deficiencies, respectively. A specialization of this generating function allows us to derive explicit formulas for the number of 321-avoiding permutations of length $n$ with precisely $k$ descents, addressing an issue brought up in the recent work of Reifegerste [21].

In Section 3 we enumerate the cyclic arrangements of length $n$ avoiding a given pattern, for all three patterns of length 4 (this is the first interesting case). Of these, two are reducible to the two cases of classical simultaneous avoidance dealt with in Section 2, and are thus tied to non-decreasing and simple Dyck paths respectively, while the third admits a bijective solution (the wraparound map) in terms of what we call non-bisecting subsets of $I_{n}$, or equivalently Grassmannian permutations, which (incidentally) underlie all three sections. The wraparound map also has an unexpected link to classical simultaneous avoidance: it establishes a one-to-one corresponce between the subsets of $I_{n}$ and the $\{132,312\}$-avoiding permutations of $[n+1]$. 
In Section 4 we also settle the enumeration of orbits of length $n$ avoiding a given orbit of length up to 4. It turns out that there is only one interesting case here, and this is still connected to simple Dyck paths, but the equivalence relation makes matters more complicated. Our approach is based on the orbit-counting lemma and this leads us to consider a class of permutations, which we refer to as affine permutations, that constitute a subgroup of the symmetric group within which the usual composition of permutations can be broken down into composition of "smaller" functions and multiplication in the group of invertible elements modulo a small integer.

\subsection{Technical preliminaries}

We denote by $\mathbb{Z}$ the set of all integers. An interval is a set $A \subseteq \mathbb{Z}$ with the property that whenever the integers $a, b, c$ satisfy $a, c \in A, a<b<c$, then $b \in A$. For integers $r$, $s$, we denote by $[r, s]$ the interval whose smallest and largest elements are $r$ and $s$ respectively. If $r>s,[r, s]$ is empty. When $r=1$, we omit it from our notation and write simply $[s]$ (thus $[s]=I_{s}$ as defined in the introduction). Also, if $r=0,[r]$ is empty. The notation $\left\{a_{1}<a_{2}<\cdots<a_{k}\right\}$ stands for the set of integers $\left\{a_{1}, a_{2}, \ldots, a_{k}\right\}$ with $a_{1}<a_{2}<\cdots<a_{k}$.

For a non-negative integer $n$, a permutation of $[n]$ is a bijection of $[n]$ to itself; $n$ is the length of the permutation. For convenience we allow the "empty" permutation, of length 0 . The set of permutations of length $n$ is denoted by $\mathcal{S}_{n}$. The notation $a_{1} a_{2} \cdots a_{n}$, which we have already tacitly used above, represents the function (almost always a permutation) which sends $i$ to $a_{i}$, e.g. 53412 is the permutation which maps 1, 2, 3, 4, 5 to 5, 3, 4, 1, 2 respectively. When necessary, we shall separate the entries with a dot, e.g. $15 \cdot 1 \cdot 12$. We shall extend this notation in the following way: if $\sigma, \tau$ are functions on $[m],[n]$ respectively, $\sigma \mid \tau$ indicates the function $\sigma(1) \sigma(2) \cdots \sigma(m) \tau(1) \tau(2) \cdots \tau(n)$. With reference to this notation, an entry of such a function $f$ is a pair $(i, f(i)) ; i$ is the position and $f(i)$ is the value of the entry.

An inversion of a permutation $\sigma$ of $[n]$ is a pair $\{i<j\} \subseteq[n]$ with $\sigma(i)>\sigma(j)$, i.e. an occurrence of the pattern 21. A descent of $\sigma$ is a point $k \in[n-1]$ such that $\sigma(k)>\sigma(k+1)$.

For the sake of completeness, we also include here the standard definition of a cyclic order (see, for example, [14]). A cyclically ordered set is a set $X$ equipped with a ternary relation $S$ such that:

$$
\begin{gathered}
\left.\begin{array}{c}
a \neq b \neq c \neq a \\
(a, b, c) \notin S
\end{array}\right\} \Leftrightarrow(c, b, a) \in S \\
\text { - }(a, b, c) \in S \Rightarrow(b, c, a) \in S \\
\text { - } \left.\begin{array}{l}
(a, b, c) \in S \\
(a, c, d) \in S
\end{array}\right\} \Rightarrow(a, b, d) \in S .
\end{gathered}
$$


Figure 1: A non-decreasing panoramic Dyck path with four valleys, one hilltop and four mountain-tops, the corresponding escalating Dyck path, and the action of the first-return and the sink-or-float bijections.

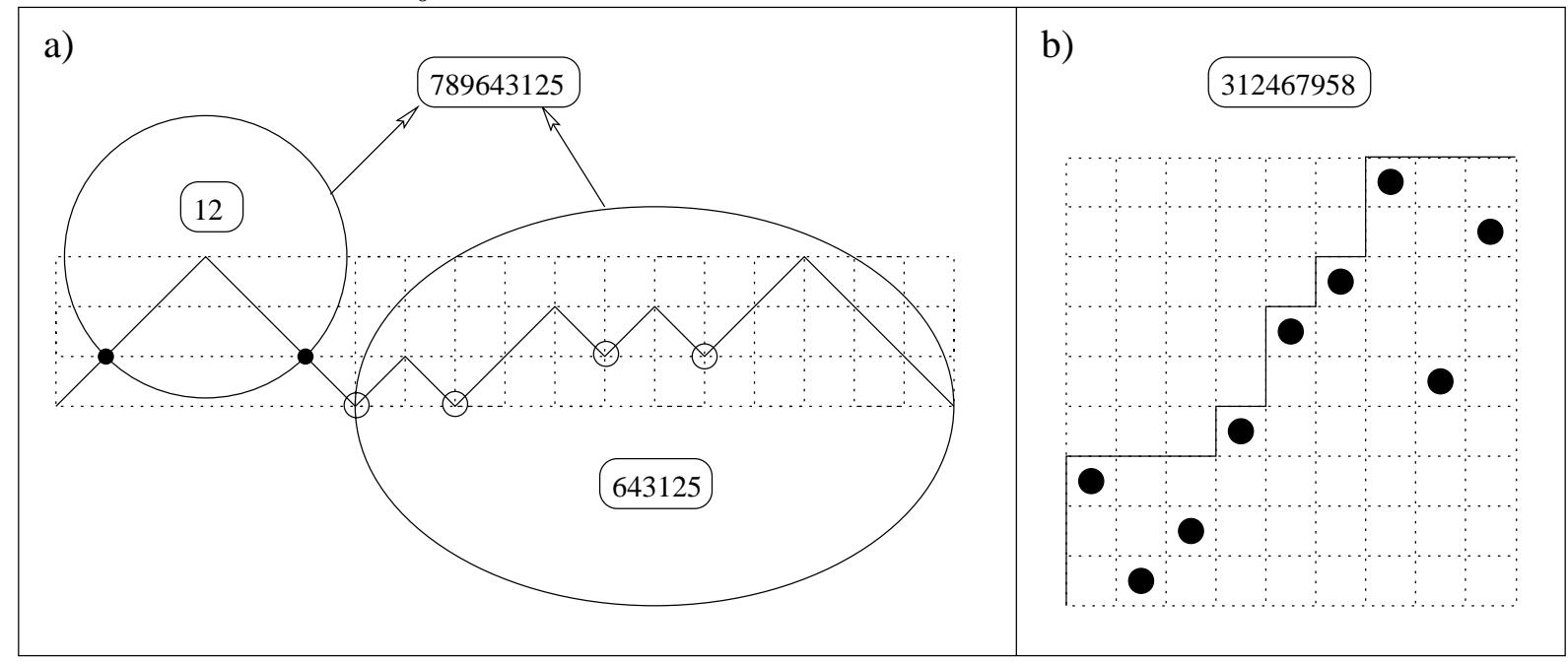

\section{Dyck paths and classical permutation avoidance}

A panoramic Dyck path of semilength $n$ is a path in the integer plane consisting of $2 n$ steps of type $u=(1,1)$ and $d=(1,-1)$, starting at the origin, ending on the $x$-axis and never going strictly below the $x$-axis. We call steps of type $u$ upward and steps of type $d$ downward. An escalating Dyck path of semilength $n$ is a path in the integer plane consisting of steps of type $v=(0,1)$ and $h=(1,0)$ starting at the origin, ending at $(n, n)$ and never going below the diagonal $x=y$. We call steps of type $v$ vertical and steps of type $h$ horizontal. A two-dimensional representation of a Dyck path in the integer plane is reminiscent of a mountainous landscape in the case of panoramic Dyck paths (Figure 1a)) and a staircase in the escalating case (Figure 1b)).

Clearly changing $u$ 's to $v$ 's and $d$ 's to $h$ 's gives a bijection between escalating and panoramic Dyck paths preserving semilength. An edge of a Dyck path is a maximal subpath consisting of steps of the same kind. An edge is upward, downward, horizontal or vertical according to the kind of step which it consists of. Edges correspond to maximal straight lines in the diagrammatic representation of Dyck paths. An edge is long if it consists of at least two steps.

Dyck paths can also be represented as strings on the alphabet $\{u, d\}$ or $\{h, v\}$. In terms of this representation, a non-empty panoramic Dyck path can be written uniquely as $u w_{1} d w_{2}$ where $w_{1}$ and $w_{2}$ are themselves (possibly empty) panoramic Dyck paths. This is known as the first-return decomposition of the Dyck path, since the $d$ corresponds to the first downward step which touches the $x$-axis. Also, $w_{1}$ and $w_{2}$ will be referred to respectively as the left and right parts of the Dyck path. 


\subsection{Non-decreasing Dyck paths and simultaneous avoidance of 132 and 3241}

\subsubsection{The first-return bijection}

Dyck paths have been the subject of much research, in particular in connection with pattern avoidance. Here we briefly describe a construction which gives a bijection between panoramic Dyck paths of semilength $n$ and 132-avoiding permutations of length $n$. This bijection is essentially the same as the one given by Krattenthaler in [15], although he gives a different, non-recursive, definition. He states that it was also discovered, independently and at the same time, by Emeric Deutsch. Our construction is the inverse of the one given in [6].

To an arbitrary panoramic Dyck path of semilength $n \geq 1$ with first-return decomposition $u w_{1} d w_{2}$, we associate a 132-avoiding permutation $\mathcal{R}(P)=\alpha|n| \beta$ with $\beta=\mathcal{R}\left(w_{2}\right)$ and $\alpha$ order-isomorphic to $\mathcal{R}\left(w_{1}\right)$ (i.e. giving an occurrence of $\mathcal{R}\left(w_{1}\right)$ using the symbols $n_{2}+1, n_{2}+2, \ldots, n-1, n_{2}$ being the semilength of $\left.w_{2}\right)$. For $n=0, \mathcal{R}$ takes the unique empty panoramic Dyck path to the unique empty permutation.

See Figure 1a) for an illustration of the action of the map $P \mapsto \mathcal{R}(P)$. This map gives a bijection between panoramic Dyck paths of semilength $n$ and 132-avoiding permutations of $[n]$. We shall refer to it as the first-return bijection.

\subsubsection{Non-decreasing Dyck paths and the zigzag construction}

Given a panoramic Dyck path, a peak is an up-step followed by a down-step, and a valley is a down-step followed by an up-step. The height of a peak/valley is the $y$-coordinate of the point common to both steps. A peak is a hilltop if has height 1, a mountain-top otherwise.

A panoramic Dyck path is non-decreasing if the heights of its valleys (left to right) form a non-decreasing sequence. Now a panoramic Dyck path always starts with an upward edge and, assuming it has $k$ valleys, is completely determined by the sequence of lengths of the first $2 k$ edges as we move from left to right (excluding the last upward and the last downward edge). We describe a procedure based on this fact to construct a set of positive integers of even cardinality from a non-decreasing Dyck path. This procedure is also illustrated in Figure 2.

A vertex of a Dyck path is simply a point on the integer lattice occupied by the path. Given an edge consisting of $x$ steps, there are precisely $x+1$ vertices lying on the edge. Starting from an arbitrary non-decreasing Dyck path $P$, we label the vertices lying on upward edges, starting with label 1 , moving left to right and increasing the label by one at each successive vertex. Then we define $a_{2 i}$ to be the label of the $i$-th peak, for $i \in[1, k]$. Clearly $\left(a_{2 i}\right)_{i=1 \ldots k}$ is a non-decreasing sequence of positive integers; indeed, if we set $a_{0}=0$, then $b_{i}=a_{2 i}-a_{2 i-2}-1$ is the length of the $i$-th upward edge, which is of course strictly positive. Hence we have $a_{2 i}-a_{2 i-2} \geq 2$, that is, there must be at least one integer in between $a_{2 i-2}$ and $a_{2 i}$. In order to uniquely characterize $P$, we also need to encode the length of the downward edges, and we would like to do so by "filling in" 
Figure 2: The zigzag construction.

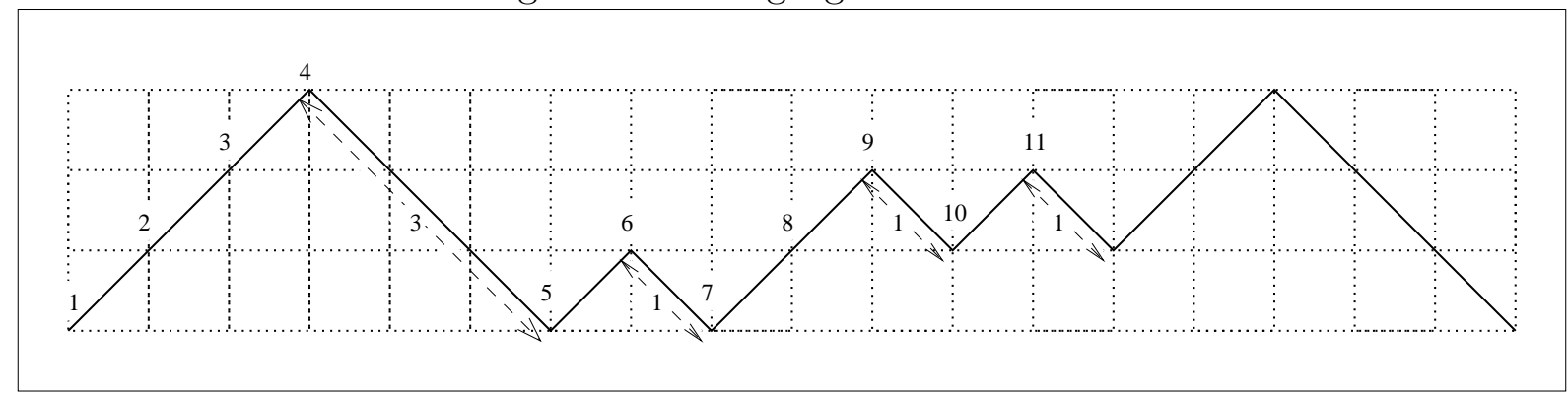

these gaps.

Since $P$ is non-decreasing, the $i$-th downward edge is no longer than the $i$-th upward edge, and of course consists of at least one step. Thus the length $c_{i}$ of the $i$-th downward edge can be anything in between 1 and $b_{i}$, the upper bound being precisely the number of integers between $a_{2 i-2}$ and $a_{2 i}$. So for $i \in[0, k-1]$ we set $a_{2 i+1}=a_{2 i}+c_{i+1}$ so that

$$
a_{2 i+1}=a_{2 i}+c_{i+1} \leq a_{2 i}+b_{i+1}=a_{2 i}+\left(a_{2 i+2}-a_{2 i}-1\right)=a_{2 i+2}-1<a_{2 i+2}
$$

and of course $a_{2 i}<a_{2 i+1}$.

Finally, note that the labelling process gives precisely one label per upward step, except for an extra label for every upward edge, corresponding to the initial vertex. Since $P$ has $k$ valleys and $k+1$ upward edges, at least one upward step comes after the $k$-th peak, so if $P$ has semilength $n$ (which is also the total number of upward steps), the label $a_{2 k}$ can be at most $(n-1)+k$. Thus $\left\{a_{1}<a_{2}<\cdots<a_{2 k}\right\}$ is a subset of $[n+k-1]$ of cardinality $2 k$. The reader can easily check that the subset corresponding to the non-decreasing Dyck path of Fig. 2 is $\{3,4,5,6,7,9,10,11\}$.

We shall refer to the map that associates this subset to the Dyck path $P$ as the zigzag construction. Observe that given arbitrary integers $b_{i}, c_{i}$ with $c_{i} \leq b_{i}(i \in[k])$ and $\sum_{i=1}^{k} b_{i}<n$, the lattice path consisting of upward and downward steps and starting at the origin with $b_{i}, c_{i}$ as the length of the $i$-th upward (respectively downward) edge can always be completed to a non-decreasing Dyck path of semilength $n$ with $k$ valleys in a unique fashion. It is now a routine matter to verify that the zigzag construction is in fact a bijection. We thus have the following proposition.

2.1 Proposition: The zigzag construction maps non-decreasing Dyck paths with precisely $k$ valleys bijectively onto subsets of cardinality $2 k$ of $[n+k-1]$.

2.2 Corollary: For a fixed integer $k$, the number of non-decreasing Dyck paths with $k$ valleys is $\left(\begin{array}{c}n+k-1 \\ 2 k\end{array}\right)$.

For a non-negative integer $i$, let $\mathrm{F}_{i}$ denote the $i$-th Fibonacci number, defined inductively by $F_{0}=0, F_{1}=1$ and $F_{i+2}=F_{i}+F_{i+1}$. Then we have that 
2.3 Corollary: The number of non-decreasing Dyck paths of semilength $n$ is the Fibonacci number $\mathrm{F}_{2 n-1}$.

Proof: A non-decreasing Dyck path of semilength $n$ can have anything between 0 and $n-1$ valleys. So the total number of non-decreasing Dyck paths of semilength $n$ is

$$
\sum_{k=0}^{n-1}\left(\begin{array}{c}
(n-1)+k \\
2 k
\end{array}\right)
$$

It is well-known (see [28]) and easy to verify that the sum of the "shallow diagonal" of Pascal's triangle starting with $\left(\begin{array}{l}s \\ 0\end{array}\right)$ gives the Fibonacci number of index $2 s+1$.

Corollary (2.3) was first proved by Barcucci et al. in [3], but the refinement in terms of valleys, although deducible from their generating functions, is not made explicit in their note. Also, this result can be inferred from Theorem 2.2 of [4], because non-decreasing Dyck paths of semilength $n$ are in bijection with directed column-convex polyominoes of area $n$, (see [11]; surprisingly, this is not mentioned in [3] in spite of the authors' paper [4]). Under this bijection, the peaks of a non-decreasing Dyck path correspond to the columns of the polyomino.

\subsubsection{Simultaneous avoidance of 132 and 3241}

In this section we show that among the 132-avoiding permutations, those which also avoid 3241 correspond, via the first-return bijection, precisely to the non-decreasing Dyck paths. First we give a simple characterization of $\{132,3241\}$-avoiding permutations.

Given a permutation $\sigma:[n] \rightarrow[n]$, a run is a maximal interval $T \subseteq[n]$ such that $\left.\sigma\right|_{T}$ is increasing. For example, the runs of 83724615 are [1], [2,3], [4,6], and [7,8]. Note that the domain $[n]$ can always be partitioned into runs. If $T=[a, b]$ is a run and $b<n$, then $T$ is nonfinal. A run $T=[a, b]$ is contiguous if $\sigma(b)-\sigma(a)=b-a$.

2.4 Theorem: A permutation $\sigma$ is $\{132,3241\}$-avoiding if and only if all the nonfinal runs of $\sigma$ are contiguous.

Proof: Assume $\sigma$ avoids $\{132,3241\}$. Then $\sigma^{-1}(1)$ is in the last run since otherwise we have a 132 pattern. If $\sigma(1)=1$, then $\sigma$ is the identity and we have no nonfinal runs. If $\sigma(1) \neq 1$, let $a<c$ be in the same nonfinal run (with $\sigma(a)<\sigma(c)$ ). If $\sigma(a)<\sigma(b)<\sigma(c)$ for some $b$, then $\sigma(b)$ cannot be to the right of $\sigma(c)$ since otherwise $\{a<c<b\}$ is an occurrence of 132. Similarly, $\sigma(b)$ cannot be to the left of $\sigma(a)$ since otherwise $\{b<a<$ $\left.c<\sigma^{-1}(1)\right\}$ is an occurrence of 3241. So we must have $a<b<c$; hence, each nonfinal run is contiguous.

Conversely, assume that all nonfinal runs of $\sigma$ are contiguous and, by way of contradiction, let $\{a<b<c\}$ be an occurrence of 132. Then $b$ cannot be in the last run. Moreover, since each value of a nonfinal run is smaller than each value of the previous run, $a$ and $b$ are in the same run. But then this run cannot be contiguous since 
$\sigma(a)<\sigma(c)<\sigma(b)$ and $\sigma(c)$ is to the right of $\sigma(b)$. Now, again by way of contradiction, suppose that $\{a<b<c<d\}$ is an occurrence of $3241(\sigma(d)<\sigma(b)<\sigma(a)<\sigma(c))$. As before, $c$ cannot be in the last run. Both $a$ and $b$ have to be in the same run as $c$. But then this run contains $\{a<b<c\}$, an occurrence of 213, and so cannot be contiguous.

It is easy to see that the first-return bijection takes the valleys of a panoramic Dyck path bijectively to the descents of the corresponding permutation $\sigma$; more precisely, the $k$-th descent at position $i$ corresponds to the $k$-th valley at height $h_{i}$, where $h_{i}=\mid\{j>$ $i \mid \sigma(j)>\sigma(i)\} \mid$, as defined in [15]. Using this fact we obtain the main result of this section.

2.5 Theorem: Under the first-return bijection of panoramic Dyck paths to 132-avoiding permutations, non-decreasing Dyck paths correspond bijectively to those permutations which also avoid 3241.

Proof: Let $i, j$ be two descents of a $\{132,3241\}$-avoiding permutation $\sigma$. In view of (2.4), $\sigma(i)>\sigma(j)$ and only the last run contributes to $h_{i}$ and $h_{j}$, implying $h_{j} \geq h_{i}$. Hence the panoramic Dyck path corresponding to $\sigma$ is non-decreasing.

Conversely, suppose the Dyck path corresponding to $\sigma$ is non-decreasing. Since $\sigma$ is 132-avoiding, whenever $i<j$ belong to the same nonfinal run and $\sigma(i)<x<\sigma(j), x$ cannot be to the right of $\sigma(j)$, since this would lead to an occurrence of 132, and neither can it be to the left of $\sigma(i)$, because then, choosing $a, b$ to be respectively the last descent before $i$ and the first after $j$, we would have $\{k>b \mid \sigma(k)>\sigma(b)\} \cup\{b\} \subseteq\{k>a \mid \sigma(k)>\sigma(a)\}$, implying $h_{j}<h_{i}$, a contradiction. So $x$ lies in between $\sigma(i)$ and $\sigma(j)$, and all nonfinal runs must be contiguous.

2.6 Corollary: The number of $\{132,3241\}$-avoiding permutations of $[n]$ with precisely $k$ descents is $\left(\begin{array}{c}n+k-1 \\ 2 k\end{array}\right)$.

Proof: Follows from (2.5) and (2.2).

From (2.5) and (2.3) we obtain the following result of West [29].

2.7 Corollary: The $\{132,3241\}$-avoiding permutations of $[n]$ are enumerated by the Fibonacci numbers $\mathrm{F}_{2 n-1}$.

\section{$2.2 \quad$ Permutations avoiding 321}

\subsubsection{The sink-or-float bijection}

We now describe a bijection that associates to an escalating Dyck path a 321-avoiding permutation. Again, this construction is essentially the same as the one given by Krattenthaler [15], who states that it was also discovered independently and at the same time by Emeric Deutsch. Our formulation is closer to the one given by Elizalde [12]. 
Given an escalating Dyck path of semilength $n$, we consider the area in the integer lattice "enclosed" by the Dyck path, the horizontal axis, and a vertical line at a distance of $n$ from the origin. There are $n$ columns in this region, and in each column precisely one horizontal step. We call a horizontal step floating if it is the first step of the edge it belongs to, and sinking otherwise. There are also precisely $n$ rows in the region under consideration.

We single out one tile per row and per column in the region, in the following manner: proceeding column by column from left to right, we choose the highest tile if the horizontal step is a floating step, and the tile in the lowest free row if the horizontal step is a sinking step. Now the required permutation associates to $i$ the height of the chosen tile in column $i$. See Figure 1b) for an example.

This construction gives a bijection between escalating Dyck paths and 321-avoiding permutations; we shall refer to it as the sink-or-float bijection and, given an escalating Dyck path $P$, we shall denote by $\operatorname{SoF}(P)$ the corresponding permutation. The bijection given by Krattenthaler actually associates a panoramic Dyck path to a 123-avoiding permutation, as opposed to a 321-avoiding permutation; given $\sigma_{1} \sigma_{2} \ldots \sigma_{n}=\sigma=\operatorname{SoF}(P)$, the panoramic Dyck path corresponding to the 123 -avoiding permutation $\sigma_{n} \sigma_{n-1} \ldots \sigma_{1}$ via Krattenthaler's bijection can be obtained from $P$ by rotating clockwise by $\pi / 4$, reflecting in a vertical line and translating horizontally (so as to start at the origin) to obtain a panoramic Dyck path.

Krattenthaler's construction goes from permutations to panoramic Dyck paths; in order to make the connection to his formulation more explicit, we now describe the inverse of SoF in terms more akin to his. Given a permutation $\sigma$, a left-to-right maximum is an integer $i \in[n]$ such that for all positive $j<i, \sigma(j)<\sigma(i)$. If $\sigma=a_{1} a_{2} \ldots a_{n}$ is 321-avoiding with left-to-right maxima $i_{1}<i_{2}<\cdots<i_{s}$, then setting $a_{0}=i_{0}=0, i_{s+1}=n+1$ and taking, for $j=1 \ldots s, b_{j}=a_{i_{j}}-a_{i_{j-1}}$ and $c_{j}=i_{j+1}-i_{j}$ respectively as the lengths of the $j$-th vertical and horizontal edges gives the escalating Dyck path corresponding to $\sigma$. Thus, in Krattenthaler's terminology, the length of a horizontal edge is one more than the length of the corresponding substring in between successive left-to-right maxima and the length of a vertical edge is the difference in value of $\sigma$ on successive maxima (with the convention $\left.\sigma(0)=a_{0}=0\right)$.

\subsubsection{Grassmannian permutations and permutation statistics}

Following Lascoux and Schützenberger [17], we shall refer to permutations with at most one descent as Grassmannian permutations. It is easy to construct a Grassmannian permutation starting from an arbitrary subset $A$ of $[n]$ : simply write all elements of $A$ in increasing order, followed by all elements of its complement in increasing order. Then if $A$ is empty, or else an interval containing 1 , the result is always the identity permutation, but this construction is otherwise injective. In fact, if we call a proper subset of $[n]$ bisecting whenever it is of the form $[k]$ with $0 \leq k<n$, we have that this construction gives a bijection between the set of non-bisecting subsets of $[n]$ and Grassmannian permutations of $[n]$. This also makes it clear that the number of such permutations is $2^{n}-n$. 
Given functions $\mathrm{w}: A \rightarrow \mathbb{Z}, \mathrm{f}: A \rightarrow \mathbb{Z}^{s}$, the statistic on $A$ of $\mathrm{f}$ with respect to $\mathrm{w}$ is the function on $\mathbb{Z}^{2}$ which associates to $(n, p) \in \mathbb{Z}^{s+1}$ the cardinality of the set $\{a \in A \mid \mathrm{w}(a)=n, \mathrm{f}(a)=p\}$. Typically for us $A$ will be a set of permutations or a set of Dyck paths and w will be the length of the permutation or the semilength of the path. There are various functions on the set of all permutations whose statistics with respect to length have been well-studied. Most of these count the number of points of a generic permutation $\sigma$ of a certain kind; we shall be interested in the following:

$\begin{array}{llll}\operatorname{exc}(\sigma) & \text { excedances } & \operatorname{fix}(\sigma) & \text { fixed points } \\ \operatorname{suff}(\sigma) & \text { sufficiencies } & \operatorname{def}(\sigma) & \text { deficiencies } \\ \operatorname{des}(\sigma) & \text { descents } & \operatorname{ides}(\sigma) & \operatorname{dips} \\ \operatorname{lt} \operatorname{trmx}(\sigma) & \text { left-to-right maxima. } & & \end{array}$

A point $i \in[n]$ is a sufficiency of a permutation $\sigma \in \mathcal{S}_{n}$ if $\sigma(i) \geq i$, and a deficiency otherwise. Sufficiencies are distinguished into excedances and fixed points according to whether the inequality is strict or not. A dip is a point $i \in[n-1]$ such that $\sigma(i)-1$ occurs to the right of $i$.

It is easy to see that $i$ is a dip of $\sigma$ if and only if $\sigma(i)-1$ is a descent of $\sigma^{-1}$; this accounts for the (standard) notation ides. Thus the number of dips of a permutation is equal to the number of descents of its inverse. We shall refer to permutations with at most one dip as monodipic permutations; note that they are the inverses of Grassmannian permutations.

We shall also consider the following functions which count the number of "features" of a certain kind of a generic Dyck path, and their statistics with respect to the semilength of the path:

$\begin{array}{llll}\operatorname{hor}(P) & \text { horizontal edges } & \operatorname{lhor}(P) & \text { long horizontal edges } \\ \operatorname{ver}(P) & \text { vertical edges } & \operatorname{lver}(P) & \text { long vertical edges } \\ \operatorname{vall}(P) & \text { valleys } & \operatorname{peak}(P) & \text { peaks } \\ \text { hill }(P) & \text { hilltops } & \operatorname{mnt}(P) & \text { mountain-tops } \\ \operatorname{sink}(P) & \text { sinking steps. } & & \end{array}$

We shall capitalize the initial letter in the notation for these functions to indicate the corresponding statistic, e.g. Ltrmx is the statistic of Itrmx. Moreover, whenever the statistic is taken over a strict subset of the domain, we shall specify this with a subscript. Thus, if $A$ is the set of $\{132,3241\}$-avoiding permutations, the statement of Corollary 2.6 can be rephrased succinctly as $\operatorname{Des}_{A}(n, k)=\left(\begin{array}{c}n+k-1 \\ 2 k\end{array}\right)$. Furthermore, we shall concatenate notation with a vertical bar to indicate joint statistics, e.g. Des|Ides indicates the statistic of the function $\sigma \mapsto \operatorname{des} \mid \operatorname{ides}(\sigma)=(\operatorname{des}(\sigma)$, ides $(\sigma))$. Finally, we shall capitalize the whole symbol to indicate the corresponding generating function, e.g. $\operatorname{DES} \mid \operatorname{IDES}(x, y, z)$ is the formal power series in $x, y, z$ in which the coefficient of the term $x^{n} y^{m} z^{t}$ equals Des $\mid \operatorname{Ides}(n, m, t)$. Thus, the first variable will always correspond to a distinguished weight (for us, typically the length or semilength), which is suppressed in the notation, and the others to the other weights according to the order in which they 
are listed. We immediately see that the following equations hold:

$$
\mathrm{fix}+\mathrm{exc}=\text { suff } \text { hor }=\text { ver }=\text { peak } \text { Lhor }=\text { Lver } \text { peak }=\text { vall }+1=\text { hill }+ \text { mnt } .
$$

Note that Ihor and Iver are not equal. We propose to use the statistics on the intuitively more manageable Dyck paths to gain results regarding the statistics on the set $\mathcal{Z}$ of 321-avoiding permutations. Statistics on $\mathcal{Z}$ were studied by Reifegerste [21, 22], Robertson et al. [23], Adin and Roichman [1] and Elizalde [12], while Krattenthaler [15] considered statistics on 123-avoiding permutations which can be trivially translated into statistics on 321-avoiding permutations.

Consideration of the sink-or-float bijection leads to the following remarks.

- As we move from left to right, we choose a tile below its predecessor precisely at the first sinking step of each horizontal edge; this gives a natural one-to-one correspondence between long horizontal edges and descents.

- For columns with sinking steps, the row below the chosen one has already been previously occupied, and if we associate a floating step to the vertical edge immediately preceding it, we see that for columns with floating steps, the row immediately below the chosen one is picked in the previous column if the corresponding vertical edge is short, and later otherwise. This gives a natural one-to-one correspondence between long vertical edges and dips.

- A horizontal step gives a tile strictly below the diagonal if and only if it is a sinking step, and if we associate a floating step to the peak immediately preceding it (switching to the panoramic perspective) we see that floating steps distinguish between fixed points (tiles on the diagonal) and excedances according to whether the corresponding peak is a hilltop or a mountain-top. The construction also makes it clear that horizontal steps give left-to-right maxima if and only if they are floating steps. This gives natural one-to-one correspondences between peaks, sufficiencies and left-to-right maxima, hilltops and fixed points, mountain-tops and excedances and sinking steps and deficiencies.

These remarks translate into the following equations:

$$
\begin{aligned}
\forall P \in \mathcal{D}: \quad & \operatorname{peak}(P)=\operatorname{suff}(\operatorname{SoF}(P))=\operatorname{trmx}(\operatorname{SoF}(P)) \\
& \operatorname{hill}(P)=\operatorname{fix}(\operatorname{SoF}(P)) \\
& \operatorname{mnt}(P)=\operatorname{exc}(\operatorname{SoF}(P)) \\
& \operatorname{sink}(P)=\operatorname{def}(\operatorname{SoF}(P)) \\
& \operatorname{lhor}(P)=\operatorname{des}(\operatorname{SoF}(P)) \\
& \operatorname{lver}(P)=\operatorname{ides}(\operatorname{SoF}(P))
\end{aligned}
$$

where $\mathcal{D}$ denotes the set of all Dyck paths. 
Note that (1) and (2) imply that 321-avoiding Grassmannian permutations correspond precisely to escalating Dyck paths with at most 1 long horizontal edge, and 321-avoiding monodipic permutations to escalating Dyck paths with at most 1 long vertical edge. We shall call these escalating Dyck paths horizontally simple and vertically simple respectively, while a path will be simple if it is one or the other.

Now any occurrence $\{x<y<z\}$ of 321 in a permutation is such that $\{x, y\}$ and $\{y, z\}$ are inversions. It is easy to see that if $\{i<j\}$ is an inversion of a permutation $\sigma$, then there must be a descent $a$ and a dip $b$ with $i \leq a<j$ and $\sigma(i) \geq \sigma(b)>\sigma(j)$, so in fact all Grassmannian permutations and all monodipic permutations are 321-avoiding. We summarize with the following proposition.

2.8 Proposition: The sink-or-float bijection maps horizontally simple escalating Dyck paths bijectively to Grassmannian permutations and vertically simple escalating Dyck paths bijectively to monodipic permutations.

\subsubsection{Simultaneous avoidance of 321 and 2143}

Just as in section 2.1 the non-decreasing Dyck paths gave us the permutations which simultaneously avoid 132 and 3241, here simple Dyck paths correspond to \{321,2143\}avoiding permutations. Note that 2143-avoiding permutations are often referred to as vexillary permutations. For the purposes of the following proof, we define a gaping step of an escalating Dyck path to be a vertical step which is not the last step of the vertical edge it belongs to.

2.9 Theorem: Under the sink-or-float bijection of escalating Dyck paths to 321-avoiding permutations, simple Dyck paths correspond bijectively to those permutations which also avoid 2143.

Proof: First we show that if an escalating Dyck path $P$ has at least two long horizontal edges and at least two long vertical edges then $\sigma$, the corresponding 321-avoiding permutation, has an occurrence of 2143. Let $e_{1}$ be the first long (vertical) edge, $s_{1}$ the first floating step immediately after $e_{1}, s_{2}$ the first sinking step (after $s_{1}$ ), $e_{2}$ the last long (horizontal) edge, $s_{3}$ the floating step of $e_{2}$ and $s_{4}$ the last sinking step (of $e_{2}$ ). For $i \in[1,4]$, we also denote by $a_{i}$ the position (column) of $s_{i}$. We claim that $\left\{a_{1}, a_{2}, a_{3}, a_{4}\right\}$ is an occurrence of 2143 .

By definition of the $s_{i}$ 's, we have $a_{1}<a_{2}$ and $a_{3}<a_{4}$ (note that $s_{3}$ and $s_{4}$ belong to the same horizontal edge); since there are at least two long horizontal edges and $s_{2}$ and $s_{3}$ belong respectively to the first and last of these, we also have $a_{2}<a_{3}$.

Now all edges before $e_{1}$ are short, meaning that there are only fixed points before $a_{1}$; since $e_{1}$ is long, $a_{1}$ is an excedance, and the row corresponding to the first gaping step of $e_{1}$ lies below the tile chosen in column $a_{1}$, and will be taken precisely at the first sinking step after $s_{1}$, i.e. $s_{2}$. Thus $\sigma\left(a_{1}\right)-\sigma\left(a_{2}\right)=\left|e_{1}\right|-1>0$. Since the tile chosen in column $a_{3}$ is immediately below $e_{2}$, and the one chosen in column $a_{4}$ is also below $e_{2}$, we also have $\sigma\left(a_{3}\right)>\sigma\left(a_{4}\right)$. To prove the claim, all that needs to be shown is that $\sigma\left(a_{4}\right)>\sigma\left(a_{1}\right)$. 
Note that the total number of sinking steps is equal to the total number of gaping steps, and that $e_{1}$ contains precisely $\left|e_{1}\right|-1$ gaping steps. Since there are at least two long vertical edges, the total number of gaping steps, and therefore of sinking steps, is at least $\left|e_{1}\right|$. But $s_{2}, s_{4}$ are respectively the first and last sinking steps, so there must be at least $\left|e_{1}\right|-2$ sinking steps between them. Moreover, the entries corresponding to floating steps constitute a strictly increasing sequence, so $\sigma\left(a_{4}\right) \geq \sigma\left(a_{2}\right)+\left(\left|e_{1}\right|-1\right)=$ $\sigma\left(a_{2}\right)+\left(\sigma\left(a_{1}\right)-\sigma\left(a_{2}\right)\right)=\sigma\left(a_{1}\right)$, and of course the inequality must be strict.

Conversely, suppose that the permutation $\sigma$ corresponding to the Dyck path $P$ has an occurrence $\{i<j<k<\ell\}$ of 2143. Then the inversion $\{i<j\}$ forces a descent $x_{1} \in[i, j-1]$ and a dip $y_{1}$ with $\sigma\left(y_{1}\right) \in[\sigma(j)+1, \sigma(i)]$, and the inversion $\{k<\ell\}$ forces a descent $x_{2} \in[k, \ell-1]$ and a dip $y_{2}$ with $\sigma\left(y_{2}\right) \in[\sigma(\ell)+1, \sigma(k)]$. Since $j<k, x_{1} \neq x_{2}$, and since $\sigma(i)<\sigma(\ell), y_{1} \neq y_{2}$. Thus by Equations (1) and (2) $P$ has at least two long vertical edges and at least two long horizontal edges.

2.10 Corollary: The $\{321,2143\}$-avoiding permutations are precisely the Grassmannian permutations and their inverses. The number of such permutations of $[n]$ is $2^{n+1}-$ $\left(\begin{array}{c}n+1 \\ 3\end{array}\right)-2 n-1$.

Proof: In the light of (2.9), it is sufficient to find the number of simple Dyck paths. Except for the identity permutation, the vertically simple Dyck paths correspond to Grassmannian permutations, so there are $2^{n}-n-1$ Dyck paths with precisely 1 long vertical edge (see the introduction to Section 2.2.2). Clearly there are just as many Dyck paths with precisely 1 long horizontal edge. Now it is sufficient to count the Dyck paths with precisely one long vertical edge and one long horizontal edge. First note that in such a Dyck path, the two long edges must have the same length, say $\ell$. The Dyck path must consist of a certain number of hilltops, say $i$, before the first long (vertical) edge, a certain number $j \leq n-\ell-i$ of hilltops after the last long (horizontal) edge, and $n-\ell-i-j$ valleys in between.

Given a subset $\{i<j<k\} \subseteq[0, n]$, we can construct an escalating Dyck path of this kind by taking $i$ for the height of the base of the vertical edge, $j+1$ for the height of the top of the vertical edge, and $k-1$ for the height of the horizontal edge. This bijection shows that the number of such paths is $\left(\begin{array}{c}n+1 \\ 3\end{array}\right)$. Thus the total number of simple paths is $2\left(2^{n}-n-1\right)-\left(\begin{array}{c}n+1 \\ 3\end{array}\right)+1$.

The formula above was first obtained by Billey et al. [5] as a corollary of their work in a different, more involved framework; their proof parallels ours, but they use a different bijection which deals with a skew partition obtained from the diagram of a permutation and do not single out the class of simple Dyck paths. In [13], Eriksson and Linusson characterize $\{321,2143\}$-avoiding permutations in terms of Fulton's essential set and thus are able to rederive the formula using a combinatorial argument, again analogous. The first few terms of the sequence given by this formula are: 1, 2, 5, 13, 33, 80, 185, 411, 885, 1862, 3853, 7881, 15993, 32284, 64945, 130359. More terms are listed in entry A088921 of $[18]$. 
We conclude this section with a lemma about Grassmannian permutations that is particularly easy to prove in the context of the sink-or-float bijection.

2.11 Lemma: Let $i$ be the only descent of a permutation $\sigma$; then $i$ is an excedance and

- for $j<i, \sigma(j) \geq j$,

- for $i<j \leq \sigma(i), \sigma(j)<j$

- for $j>\sigma(i), \sigma(j)=j$.

Proof: The assertion says that a permutation with precisely one descent consists of an initial (possibly empty) sequence of fixed points, followed by a (non-empty) sequence of excedances, the last one of which is the descent $i$, a (non-empty) sequence of deficiencies ending at position $\sigma(i)$, and finally a (possibly empty) sequence of fixed points. This is evident from the fact that it is the image under the sink-or-float bijection of a Dyck path with precisely one long horizontal edge; we only observe that discarding the final tail of hilltops gives a Dyck path of semilength $\sigma(i)$.

Note that the above lemma implies in particular that there can be no excedances to the right of the only descent of a Grassmannian permutation; we shall use this fact repeatedly in the later sections.

\subsubsection{A generating function for some statistics}

In this section we use the considerations in Section 2.2.2 to obtain information about statistics on 321-avoiding permutations. We derive the generating function $F$ counting Dyck paths by semilength and by the number of hilltops, mountain-tops, sinking steps, long horizontal edges and long vertical edges, or equivalently 321-avoiding permutations by length and by the number of fixed points, excedances, deficiencies, descents and dips.

We have already seen that the sink-or-float bijection gives a one-to-one correspondence between peaks of a Dyck path and the sufficiencies (which are also left-to-right maxima) of the corresponding 321-avoiding permutation. The enumeration of Dyck paths by the number of valleys (equivalently, peaks) dates back to the work of Narayana in 1955 [19]. The solution is given by the well-known Narayana numbers; more precisely, for $n \neq$ 0 , Peak $(n, k)=N_{n, k}=\frac{1}{n}\left(\begin{array}{c}n \\ k-1\end{array}\right)\left(\begin{array}{l}n \\ k\end{array}\right)$. The corresponding generating function $\operatorname{PEAK}(x, v)$ satisfies the quadratic

$$
x X^{2}+(v x-1-x) X+1=0 .
$$

Thus we already have that

2.12 Proposition: The statistics Suff and Ltrmx are Narayana distributed over the 321-avoiding permutations. 
In order to deal with other statistics, we define a weight on a generic Dyck path by

$$
f(P)=x^{\ell(P)} y^{\operatorname{lhor}(P)} z^{\operatorname{lver}(P)} u^{\operatorname{hill}(P)} v^{\operatorname{mnt}(P)} w^{\operatorname{sink}(P)} s^{\alpha(P)} t^{\beta(P)}
$$

where $\ell(P)$ is the semilength of $P$ and $\alpha(P)$ (respectively $\beta(P)$ ) is 1 if $P$ starts (ends) with a hilltop and 0 otherwise. Note that if $G(s, t, u, v, w, x, y, z)$ denotes the formal power series $G=\sum_{P \in \mathcal{D}} f(P)$, then

$$
G(1,1, u, v, w, x, y, z)=\mathrm{HILL}|\mathrm{MNT}| \operatorname{SINK}|\operatorname{LHOR}| \operatorname{LVER}(x, u, v, w, y, z)=F,
$$

the generating function we require.

Now we observe that apart from the empty Dyck path, with weight 1, and the unique Dyck path of semilength 1, with weight stux, Dyck paths can be distinguished into those of the form $u d P^{\prime}$, with $P^{\prime}$ a non-empty panoramic Dyck path (class $\mathcal{A}$ ), and those of the form $u Q d R$, with $Q, R$ panoramic Dyck paths, $Q$ non-empty (class $\mathcal{B}$ ).

In class $\mathcal{A}$, the right part $P^{\prime}$ gives no contribution to $\alpha(P)$ and the first hilltop is certainly not the last, so $\sum_{P \in \mathcal{A}} f(P)=u s x G(1, t, u, v, w, x, y, z)$, whereas in class $\mathcal{B}$, since $Q$ is non-empty, $u Q d$ does not start with a hilltop, and so does not contribute to $\alpha$, nor to $\beta$. Moreover, $u Q d$ will have one more long upward (downward) edge than $Q$ precisely when $Q$ starts (ends) with a hilltop, and exactly the same number otherwise. Also, all peaks (whether hilltops or mountain-tops) of $Q$ become mountain-tops of $u Q d$, while both the semilength and the number of sinking steps go up precisely by one. Finally, $R$ does not contribute to $\alpha$, and we have $\sum_{P \in \mathcal{B}} f(P)=w x(G(y, z, v, v, w, x, y, z)-1) G(1, t, u, v, w, x, y, z)$.

So we conclude

$$
\begin{aligned}
G(s, t, u, v, w, x, y, z)= & 1+\operatorname{stux}+\operatorname{sux}(G(1, t, u, v, w, x, y, z)-1) \\
& +w x(G(y, z, v, v, w, x, y, z)-1) G(1, t, u, v, w, x, y, z) .
\end{aligned}
$$

Substituting first $s=t=1$, then $u=v, s=y, t=z$ and finally $s=1, t=z, u=v$ we obtain the system of three equations in three unknowns

$$
\begin{aligned}
& F=1+u x F+x F(B-1) \\
& B=1+v x y z+v x y(C-1)+x(B-1) C \\
& C=1+v x z+v x(C-1)+x(B-1) C
\end{aligned}
$$

where $B=G(y, z, v, v, w, x, y, z)$ and $C=G(1, z, v, v, w, x, y, z)$. Eliminating $B$ and $C$, we deduce that $F$ satisfies the quadratic

$$
A_{2} F^{2}+A_{1} F+1=0
$$

where

$$
\begin{aligned}
& A_{2}=-x^{2} w u-v x^{2} u+v x^{3} w u+u x^{3} v y z w-u x^{3} v y w+v x-u x \\
& +u^{2} x^{2}+v x^{2} y w+x w+v x^{2} z w-v x^{3} z w u-v x^{2} w \\
& A_{1}=-v x+2 u x+v x^{2} y z w-v x^{2} y w-v x^{2} z w+v x^{2} w-1-x w
\end{aligned}
$$


Equation (4) can be specialised to more manageable forms; substituting $u=v=w=$ $y=z=1$ we obtain the familiar functional equation $x X^{2}-X+1=0$ for the Catalan numbers; substituting $w=y=z=1$ and $u=v$ (so as not to distinguish between hilltops and mountain-tops) we obtain that $\operatorname{PEAK}(x, v)$ satifies $(3)$, as expected. Substituting $w=u=y=z$, we obtain that $\operatorname{MNT}(x, v)$ satisfies

$$
v x Y^{2}+(x-1-v x) Y+1=0 .
$$

It is easy to verify that substituting $X=v Y-v+1$, Equation (3) reduces to Equation (5); from this it follows that the coefficient of $x^{n} v^{k}$ in $\operatorname{MNT}(x, v)$ is just the coefficient of $x^{n} v^{k+1}$ in $\operatorname{PEAK}(x, v)$, except for $n=k=0$, in which case we have a 1 corresponding to the trivial Dyck path. Thus mountain-tops are also Narayana distributed. This fact has also been shown by Deutsch [9]; while the corresponding excedance statistic was shown to be Narayana distributed over the 321-avoiding permutations by Reifegerste [21]. Substituting $u=v=y=z=1$ into Equation (4) again gives Equation (5) (with $w$ for $v$ ), so the distribution of deficiencies over 321-avoiding permutations (sinking steps over all Dyck paths) is identical to that of mountain-tops, but this also follows from the fact that for any permutation $\sigma$ of $[n], \operatorname{suff}(\sigma)+\operatorname{def}(\sigma)=n$ and the symmetry of the Narayana numbers $\left(N_{n, k}=N_{n, n+1-k}\right)$. Substituting $w=z=1$ we obtain the joint distribution for fixed points, descents and excedances over 321-avoiding permutations, which was recently derived independently by Elizalde [12] (Section 3) using similar ideas. If we further substitute $y=1$ we obtain the generating function HILL |MNT, derived by Deutsch in [10] (Equation (6.12)). Finally, substituting $v=1$ gives the generating function for fixed points over the 321-avoiding permutations; however, the statistic Fix has been expressed more explicitly by Robertson et al. [23].

The general solution to Equation (4) is rather cumbersome to express explicitly. Since for any permutation $\sigma$ of $[n]$ we have that def + fix + exc $=n$, and since Lhor $=$ Lver, in the following statement, apart from summarizing the above considerations, we give the explicit solution in the cases $y=z=1$ and $u=v=w=1$.

\subsection{Theorem:}

- The generating function

$$
\begin{aligned}
F(u, v, w, x, y, z) & =\operatorname{HILL}|\operatorname{MNT}| \operatorname{SINK}|\operatorname{LHOR}| \operatorname{LVER}(x, u, v, w, y, z) \\
& =\operatorname{FIX}|\operatorname{EXC}| \operatorname{DEF}|\operatorname{DES}| \operatorname{IDES}_{\mathcal{Z}}(x, u, v, w, y, z)
\end{aligned}
$$

is the unique non-spurious solution of Equation (4).

- The statistics Mnt and Sink are Narayana distributed over Dyck paths, and the statistics Exc and Def are Narayana distributed over the 321-avoiding permutations.

- The joint statistic of excedances, fixed points and deficiencies over 321-avoiding permutations (mountain-tops, hilltops and sinking steps over Dyck paths) is given by: 


$$
\begin{aligned}
\operatorname{FIX} \mid \operatorname{EXC}_{D_{\mathcal{Z}}}(x, u, v, w) & =\operatorname{HILL}|\operatorname{MNT}| \operatorname{SINK}(x, u, v, w) \\
& =\frac{1+v x+w x-2 u x-\sqrt{(1-v x-w x)^{2}-4 v w x^{2}}}{2 x(1-u x)(v+w-u)+2 v w x^{2}}
\end{aligned}
$$

- The joint statistic of descents and dips over 321-avoiding permutations (long horizontal and long vertical edges over Dyck paths) is given by:

$$
\begin{aligned}
\operatorname{DES} \mid \operatorname{IDES}_{\mathcal{Z}}(x, y, z) & =\operatorname{LHOR} \mid \operatorname{LVER}(x, y, z) \\
& =\frac{Q-\sqrt{Q^{2}-4 P}}{2 P}
\end{aligned}
$$

where $P=x(1-x+x y)(1-x+x z)$ and $Q=1-x^{2}(y-1)(z-1)$.

Note that the generating function in Equation (6) can be expressed as $\frac{\mathrm{C}\left(P / Q^{2}\right)}{Q}$, where $\mathrm{C}(x)$ is the familiar Catalan generating function, i.e. $\mathrm{C}(x)=\frac{1-\sqrt{1-4 x}}{2 x}=\sum_{i \geq 0} \mathrm{c}_{i} x^{i}$, with $\mathrm{c}_{i}=\frac{1}{i+1}\left(\begin{array}{c}2 i \\ i\end{array}\right)$, the $i$-th Catalan number. Setting $M=1-Q=x^{2}(y-1)(z-1)$, we obtain the series

$$
\sum_{i \geq 0} \mathrm{c}_{i} P^{i}\left(\sum_{j \geq 0} M^{j}\right)^{2 i+1}
$$

and from this it is a routine matter to extract the following expression for the coefficients.

2.14 Proposition: The number of 321-avoiding permutations of length $n$ with precisely $b$ descents and $c$ dips is given by:

$$
\sum_{i \geq 0} \mathrm{c}_{i} \sum_{\begin{array}{c}
2 s+a_{1}=n \\
b_{1}+b_{2}=b \\
c_{1}+c_{2}=c
\end{array}}(-1)^{n+b+c+i}\left(\begin{array}{c}
i \\
b_{1}
\end{array}\right)\left(\begin{array}{c}
i \\
c_{1}
\end{array}\right)\left(\begin{array}{c}
s \\
b_{2}
\end{array}\right)\left(\begin{array}{c}
s \\
c_{2}
\end{array}\right)\left(\begin{array}{c}
2 i+s \\
s
\end{array}\right)\left(\begin{array}{c}
2 i-b_{1}-c_{1} \\
a_{1}-b_{1}-c_{1}-i
\end{array}\right) .
$$

The above formula is not especially enlightening, and is probably not the most concise way of expressing the coefficients, but apart from being specializable to a much less daunting, and more useful, form (as we shall see in the following section), it does make it computationally feasible to determine these numbers algorithmically. For example, of the 1583850964596120042686772779038896321 -avoiding permutations of length 60 , there are 2539791795216418415246700 which have precisely 19 descents and 5 dips.

\subsubsection{The descent statistic on 321-avoiding permutations and a refinement of the Catalan numbers}

In [21], Reifegerste studies the descent statistic on 321-avoiding permutations. She reduces the problem to an equivalent one on a certain class of Motzkin paths but does not 
give an explicit formula. Here we obtain an expression for the number of 321-avoiding permutations of length $n$ with precisely $m$ descents. In order to do this, we simply need to determine the coefficient of $x^{n} y^{m}$ in the generating function $\mathrm{C}\left(P^{\prime}\right)$ with $P^{\prime}=x(1-x+x y)$, obtained by substituting $z=1$ in (6). Routine manipulation gives the following expression for the coefficients.

2.15 Proposition: The descent statistic on 321-avoiding permutations is given by:

$$
\begin{aligned}
\operatorname{Des}_{\mathcal{Z}}(n, m) & =\sum_{i}(-1)^{n+i+m} \frac{1}{i+1}\left(\begin{array}{c}
2 i \\
n
\end{array}\right)\left(\begin{array}{c}
n \\
i
\end{array}\right)\left(\begin{array}{c}
n-i \\
m
\end{array}\right) \\
& =\sum_{i}(-1)^{n+i+m} \mathrm{c}_{i}\left(\begin{array}{c}
i \\
n-i
\end{array}\right)\left(\begin{array}{c}
n-i \\
m
\end{array}\right) .
\end{aligned}
$$

In particular, we have that

$\mathbf{A} \operatorname{Des}_{\mathcal{Z}}(2 m, m)=\mathbf{c}_{m}$

B $\operatorname{Des}_{\mathcal{Z}}(2 m+1, m)=\left(\begin{array}{c}2 m+2 \\ m\end{array}\right)$.

In the above summations, as in the next corollary, all variables are non-negative integers and we adopt the convention that $\left(\begin{array}{l}a \\ b\end{array}\right)=0$ if $a$ is negative or $b \notin[0, a]$. Parts $\mathrm{A}$ and $\mathrm{B}$ are obtained from the second and first formulas respectively by substituting $n=2 m$ and $n=2 m+1$ and simplifying. Part A is equivalent to Exercise 6.19, $q^{4}$ of [26]. Since we know that the total number of 321-avoiding permutations of length $n$ is the $n$-th Catalan number, we also have the following identity refining the Catalan numbers.

\subsection{Corollary:}

$$
\sum_{m} \sum_{i}(-1)^{n+i+m} \mathrm{c}_{i}\left(\begin{array}{c}
i \\
n-i
\end{array}\right)\left(\begin{array}{c}
n-i \\
m
\end{array}\right)=\mathrm{c}_{n} .
$$

In Table 1 we give the values of the first few of these numbers. Note that the second column in this table gives the number of permutations with precisely 1 descent, which we know to be $2^{n}-n-1$. These numbers are known as the Eulerian numbers, and appear as sequence A000295 in [18]. We remark that, for fixed $m$, it is possible to use Zeilberger's algorithm and Petkovšek's algorithm (see [20]) to obtain (hypergeometric) closed form formulas for $\operatorname{Des}_{\mathcal{Z}}(n, m)$. In particular,

- $\operatorname{Des}_{\mathcal{Z}}(n, 2)=2^{n-3} n(n-5)+\left(\begin{array}{c}n+1 \\ 2\end{array}\right)$

- $\operatorname{Des}_{\mathcal{Z}}(n, 3)=\frac{1}{3} 2^{n-6} n(n-1)\left(n^{2}-13 n+46\right)-\left(\begin{array}{c}n+1 \\ 3\end{array}\right)$

- $\operatorname{Des}_{\mathcal{Z}}(n, 4)=\frac{1}{9} 2^{n-10} n(n-1)(n-2)(n-9)\left(n^{2}-15 n+68\right)+\left(\begin{array}{c}n+1 \\ 4\end{array}\right)$. 
Table 1: A refinement of the Catalan numbers

\begin{tabular}{|c|c|c|c|c|c|c|c|c|c|}
\hline$n$ & $\begin{array}{l}m \\
0\end{array}$ & 1 & 2 & 3 & 4 & 5 & 6 & 7 & $\begin{array}{l}\text { Row } \\
\text { sum }\end{array}$ \\
\hline 2 & 1 & 1 & & & & & & & 2 \\
\hline 3 & 1 & 4 & & & & & & & 5 \\
\hline 4 & 1 & 11 & 2 & & & & & & 14 \\
\hline 5 & 1 & 26 & 15 & & & & & & 42 \\
\hline 6 & 1 & 57 & 69 & 5 & & & & & 429 \\
\hline 7 & 1 & 120 & 252 & 56 & & & & & 1430 \\
\hline 8 & 1 & 247 & 804 & 364 & 14 & & & & 4862 \\
\hline 9 & 1 & 502 & 2349 & 1800 & 210 & & & & 16796 \\
\hline 10 & 1 & 1013 & 6455 & 7515 & 1770 & 42 & & & 58786 \\
\hline 11 & 1 & 2036 & 16962 & 27940 & 11055 & 792 & & & 208012 \\
\hline 12 & 1 & 4083 & 43086 & 95458 & 57035 & 8217 & 132 & & 742900 \\
\hline 13 & 1 & 8178 & 106587 & 305812 & 257257 & 62062 & 3003 & & 2674440 \\
\hline 14 & 1 & 16369 & 258153 & 931385 & 1049685 & 381381 & 37037 & 429 & 9694845 \\
\hline
\end{tabular}

One way to obtain the general form for $\operatorname{Des}_{\mathcal{Z}}(n, m)$ is to use hypergeometric functions. The following derivation was given by Krattenthaler [16]. For a treatment of hypergeometric functions, the reader is referred to [25].

Starting from the sum

$$
\sum_{i=0}^{n-m}(-1)^{n+i+m} \frac{1}{i+1}\left(\begin{array}{c}
2 i \\
n
\end{array}\right)\left(\begin{array}{c}
n \\
i
\end{array}\right)\left(\begin{array}{c}
n-i \\
m
\end{array}\right)
$$

reversing the order of summation and writing the resulting sum in hypergeometric notation, we obtain the expression

$$
\frac{(1-2 m+n)_{n}}{m !(n-m+1) !}{ }_{3} F_{2}\left[\begin{array}{c}
m-n-1, m-\frac{n}{2}, \frac{1}{2}+m-\frac{n}{2} \\
m-n, \frac{1}{2}+m-n
\end{array}\right]
$$

where $(x)_{n}$ stands for the rising factorial $\prod_{i=0}^{n-1}(x+i)$, and where we use hypergeometric function notation, that is, for integers $p, q$, parameters $a_{1}, a_{2}, \ldots a_{p}, b_{1}, b_{2}, \ldots b_{q}$ and a variable $z$, the symbol

$$
{ }_{p} F_{q}\left[\begin{array}{c}
a_{1}, a_{2}, \ldots a_{p} \\
b_{1}, b_{2}, \ldots b_{q}
\end{array} ; z\right]
$$

denotes the function

$$
\sum_{n=0}^{\infty} \frac{\left(a_{1}\right)_{n}\left(a_{2}\right)_{n} \cdots\left(a_{p}\right)_{n} z^{n}}{\left(b_{1}\right)_{n}\left(b_{2}\right)_{n} \cdots\left(b_{q}\right)_{n} n !}
$$


We now use the contiguous relation

$$
\begin{array}{r}
{ }_{3} F_{2}\left[\begin{array}{c}
a, b, c \\
d, e
\end{array} ; z\right]=\frac{(d-1)(e-1)}{z(b-1)(c-1)}{ }_{3} F_{2}\left[\begin{array}{c}
a, b-1, c-1 \\
d-1, e-1
\end{array} ; z\right] \\
-\frac{(d-1)(e-1)}{z(b-1)(c-1)}{ }_{3} F_{2}\left[\begin{array}{c}
a-1, b-1, c-1 \\
d-1, e-1
\end{array} ; z\right]
\end{array}
$$

iteratively, to obtain the relation

$$
\begin{gathered}
{ }_{3} F_{2}\left[\begin{array}{c}
a, b, c \\
d, e
\end{array} ; z\right]=(-1)^{m} \frac{(d-m)_{m}(e-m)_{m}}{z^{m}(b-m)_{m}(c-m)_{m}}{ }_{3} F_{2}\left[\begin{array}{c}
a-m, b-m, c-m \\
d-m, e-m
\end{array} ; z\right] \\
+\sum_{k=1}^{m}(-1)^{k-1} \frac{(d-k)_{k}(e-k)_{k}}{z^{k}(b-k)_{k}(c-k)_{k}}{ }_{3} F_{2}\left[\begin{array}{c}
a-k+1, b-k, c-k \\
d-k, e-k
\end{array} ; z\right] .
\end{gathered}
$$

If we apply this relation to our series, the result simplifies to the expression

$$
\begin{aligned}
& (-1)^{m} \frac{(n+1)_{n}}{m !(n-m+1) !}{ }_{3} F_{2}\left[\begin{array}{c}
-1-n,-\frac{n}{2}, \frac{1}{2}-\frac{n}{2} \\
-n, \frac{1}{2}-n
\end{array} ; 1\right] \\
& \quad+\sum_{k=1}^{m}(-1)^{k-1} \frac{(n-2 m+2 k+1)_{n}}{m !(n-m+1) !}{ }_{2} F_{1}\left[\begin{array}{c}
-k+m-\frac{n}{2}, \frac{1}{2}-k+m-\frac{n}{2} ; 1 \\
\frac{1}{2}-k+m-n
\end{array}\right] .
\end{aligned}
$$

The ${ }_{2} F_{1}$-series can be evaluated by means of the Chu-Vandermonde summation formula (see [25], (1.7.7), Appendix (III.4))

$$
{ }_{2} F_{1}\left[\begin{array}{cc}
a,-t & ; 1 \\
c &
\end{array}\right]=\frac{(c-a)_{t}}{(c)_{t}}
$$

for any non-negative integer $t$, while the ${ }_{3} F_{2}$-series is balanced and can therefore be evaluated by means of the Pfaff-Saalschütz summation formula (see [25], (2.3.1.3), Appendix (III.2))

$$
{ }_{3} F_{2}\left[\begin{array}{c}
a, b,-t \\
c, 1+a+b-c-t
\end{array} ; 1\right]=\frac{(c-a)_{t}(c-b)_{t}}{(c)_{t}(c-a-b)_{t}}
$$

where $t$ must again be a non-negative integer. As a result we obtain

2.17 Theorem: The descent statistic on 321-avoiding permutations is given by:

$$
\begin{aligned}
\operatorname{Des}_{\mathcal{Z}}(n, m) & =(-1)^{m}\left(\begin{array}{c}
n+1 \\
m
\end{array}\right) \\
& +\sum_{k=1}^{m} \frac{(-1)^{k-1} 2^{n-2 m+2 k}(n-2 m+2 k+1)_{2 m-2 k}(n-m+2)_{k-1}}{m !(m-k) !} .
\end{aligned}
$$

Note that the number of summands in the above formula (as opposed to the one given in (2.15)) depends only on $m$; thus for arbitrary fixed $m$ this is a closed form formula in $n$ for the number of 321-avoiding permutations of $[n]$ with precisely $m$ descents. 


\section{Cyclic arrangements}

Let $n$ be a positive integer. Given an integer $k$, the notation $k$ omod $n$ stands for the unique integer in $[n]$ congruent to $k$ modulo $n$. Given integers $i, j, i \oplus j$ denotes $i+j$ omod $n$; in this section, the value of $n$ will be clear from the context. The operation $\ominus$ is defined analogously. These operations can be thought of the usual operations of modular arithmetic, except that they use the symbol $n$ in place of 0 . Now let $\epsilon_{n}, \rho_{n}$ be the permutations given by $i \mapsto i \oplus 1$ and $i \mapsto n-i+1$ respectively. Note that $\rho_{n}$ is an involution and $\epsilon_{n}^{n}$ is the identity permutation of $[n]$.

If we consider permutations as functions from $[n]=I_{n}$ to itself, with the domain equipped with the (obvious) cyclic order as constructed in Section 1, and the codomain equipped with the usual linear order, then the equivalence class of a permutation $\sigma$, under order-isomorphism as defined in Section 1, consists of all "rotations" of the permutation, that is, all permutations $\epsilon_{n}^{i} \circ \sigma$ with $i \in[0, n-1]$.

We shall call these equivalence classes cyclic arrangements of length $n$ (or simply of $[n])$; clearly there are $(n-1)$ ! cyclic arrangements of $[n]$. We shall denote the equivalence class of $\sigma$ by $(\sigma)$. Note that technically a cyclic arrangement is a set of permutations. So for example $(4312)=\{4312,3124,1243,2431\}$.

The reverse of a permutation $\sigma \in \mathcal{S}_{n}$ is the permutation $\rho_{n} \circ \sigma$; the reverse can be obtained by reading $\sigma$ "right to left", e.g. 43152 is the reverse of 25134 . The complement of a permutation $\sigma \in \mathcal{S}_{n}$ is the permutation $\sigma \circ \rho_{n}$; the complement can be obtained by subtracting the value of each entry from $n+1$, that is, swapping the largest value of $\sigma$ with the smallest one, the second largest with the second smallest, et cetera. So for example, 41532 is the complement of 25134.

For fixed $n$, reversal and complementation are involutions of the set $\mathcal{S}_{n}$. Moreover, in the context of classical pattern avoidance (i.e. only linear orders), if $r(\sigma)$ denotes the reverse of $\sigma$, or its complement, then we have that $\sigma$ has an occurrence of $\tau$ if and only if $r(\sigma)$ has an occurrence of $r(\tau)$. The same property is satisfied by one other involution of the set of permutations: the operation of taking inverses. In classical pattern avoidance, it has become standard practice to use these three operations to reduce the number of different cases to be analysed, since for any composition $p$ of the operations of reversal, complementation and taking inverses, the set of $p(\tau)$-avoiding permutations is the image under $p$ of the set of $\tau$-avoiding permutations (and in particular these two sets have the same cardinality).

For cyclic arrangements, reversal and complementation are well-defined, but not taking inverses; i.e. for any cyclic arrangement $\theta$, the set $\{r(\sigma) \mid \sigma \in \theta\}$ (where $r$ stands for complementation or reversal) is itself a cyclic arrangement, but this property fails for the operation of taking inverses. Thinking of reversal and complementation respectively as pre- and post-composition with $\rho_{n}$, we immediately see why this is true for complementation, while for reversal it follows from the fact that that $\epsilon_{n} \circ \rho_{n} \circ \epsilon_{n}=\rho_{n}$.

In this section we deal with the enumeration of cyclic arrangements of $[n]$ avoiding any fixed cyclic arrangement (pattern) of length $t$, for $t \leq 4$. Recall that, according to the definitions in Section 1, for cyclic arrangements $x, y$, the fact that $x$ avoids $y$ is equivalent 
to every member of $x$ avoiding every member of $y$ in the classical sense of permutation avoidance. For $t=1,2$, there is only one cyclic arrangement of $[t]$, so for $n \geq t$ no cyclic arrangements can avoid any pattern of length $t$. For $t=3$, there are only 2 distinct patterns, (123) and (321), which are complements (and reverses!) of each other. Hence if a permutation $\sigma$ of $[n]$ is to avoid (123), then all subsets of $[n]$ of cardinality three must be occurrences of (321). Now it is easy to see that $(\sigma)=\left(\rho_{n}\right)$, i.e. there is only one cyclic arrangement which avoids (123).

For $t=4$, there are 6 different patterns: (1243), (1342), (1324), (1423), (1234), (1432). Since 1243 is the reverse of $3421 \in(1342)$, (1324) is the reverse of $4231 \in(1423)$ and 1234 is the reverse of $4321 \in(1432)$, it is sufficient to consider only one from each of these pairs.

\subsection{The pattern (1243) and the wraparound map}

We now construct a map which associates to a subset of $[n]$ a (1243)-avoiding permutation of $[n+1]$. Although formally we prefer to think of the domain as the power set of $[n]$, the function is perhaps most effectively described in terms of binary strings of length $n$, on the alphabet $\{T, B\}$ ( $T$ for top, $B$ for bottom). Given a subset $A$ of $[n]$, consider the binary string of length $n$ having $T$ at position $i$ if and only if $i \in A$ (and $B$ otherwise). We can think of this string as a slightly modified characteristic function of $A$. Now

- label the $B$ 's of the string, starting with 1 at the rightmost $B$, moving to the left and increasing with one at each successive $B$;

- add the next label to the left of the string;

- label the $T$ 's of the string, starting with the next label at the leftmost $T$, moving to the right and increasing with one at each successive $B$.

The following example shows how we obtain the permutation 678543921 from the set $\{1,2,6\} \subseteq[1,8]:$

\begin{tabular}{|c|c|c|c|c|c|c|c|c|}
\hline & 1 & 2 & 3 & 4 & 5 & 6 & 7 & 8 \\
\hline & & & 5 & 4 & 3 & & 2 & 1 \\
\hline 6 & $T$ & $T$ & $\overleftarrow{B}$ & $\overleftarrow{B}$ & $\overleftarrow{B}$ & $T$ & $\overleftarrow{B}$ & $\overleftrightarrow{B}$ \\
\hline & $\overrightarrow{7}$ & $\overrightarrow{8}$ & & & & $\overrightarrow{9}$ & & \\
\hline
\end{tabular}

We shall refer to the above map as the wraparound map. For the purposes of the following proof, we make the following definitions. Suppose $X \subseteq[n]$ is an occurrence of the permutation $\tau$, where $\tau \in \mathcal{S}_{|X|}$ is either the identity permutation or $\rho_{|X|}$, and suppose also that $\sigma(X)$ is an interval. If $\tau$ is the identity and $n \in \sigma(X)$, then $\sigma(X)$ is an increasing 
chain, and if $\tau$ is $\rho_{|X|}$ and $1 \in \sigma(X)$, then $\sigma(X)$ is a decreasing chain. If instead $X$ is an occurrence of $(\tau)$, then $\sigma(X)$ is a cyclic increasing (decreasing) chain. Also, the chain starts at position $i$ if $\sigma(i)$ is the smallest element of $\sigma(X)$.

For example, $\{8,9\}$ and $\{4,3,2,1\}=\{1,2,3,4\}$ are respectively an increasing chain of length 2 starting at position 3 and a decreasing chain of length 4 starting at position 5 , both in the above permutation 678549321, (note the shift of one in the positions) and $\{5,6,7,8\}$ is the longest cyclic increasing chain in 37148562 (starting at position 6).

In the statement of the following theorem, we think of the image of the wraparound map as a cyclic arrangement rather than a permutation; that is, strictly speaking we compose with the map $x \rightarrow(x)$. However, for the purposes of the proof, it does matter which particular representative the wraparound map chooses.

3.1 Theorem: The wraparound map takes the set of non-bisecting ${ }^{3}$ subsets of $[n]$ bijectively onto the set of (1243)-avoiding cyclic arrangements of $[n+1]$.

Proof: First we show that the permutation obtained indeed avoids (1243). Suppose, by way of contradiction, that there is an occurrence of $(4312)=(1243)$. If the 4 does not occur as a label of a $T$, then all the labels smaller than it form a decreasing sequence to its right, and therefore cannot give an occurrence of 312. So we may assume that 4 occurs as a label of a $T$. Then all smaller labels, in particular the one occurring as 3, either are to its left or else correspond to a $B$. So 3 must occur in one of these two possible ways. In both cases, the labels smaller than the one occurring as 3 , and (cyclically) in between the occurrence of 3 and the occurrence of 4 , form a decreasing subsequence of labels corresponding to $B$ 's, and so cannot give an occurrence of 12 .

Next we show that any (1243)-avoiding cyclic arrangement can be obtained by the wraparound map. Consider an arbitrary (1243)-avoiding cyclic arrangement $\theta$ and let $C$ denote the longest cyclic increasing chain in any representative of $\theta$ (note this is independent of the particular choice of representative) and $x$ denote the smallest element (value) of $C$. We can choose the representative $\sigma$ so that $\sigma(1)=x$. Also, we denote by $A_{\theta}$ the set $\{i \in[n] \mid \sigma(i+1) \in C\}$. It is now sufficient to show that the values smaller than $x$ form a decreasing subsequence, because then $\sigma$ is the image of $A_{\theta}$ under the wraparound map: the values less than $x$ correspond to $B$ 's and the values larger than $x$ correspond to $T$ 's.

Consider any two values $a<b$ less than $x$ and suppose, by way of contradiction, that $a$ occurs to the left of $b$. Note that since $C$ is longest possible, if $x$ is at least 2 then $x-1$ must appear between the values $x$ and $n+1$. In our case, since $a<b<x, x \geq 3$. Now any two values less than $x$ appearing in between $x$ and $n+1$ must be an inversion, otherwise we would get an occurrence of 3124 with $x$ as 3 and $n+1$ as 4 . Thus, the first value less than $x$ is $x-1$. Since $a$ is to the left of $b$ and $a<b<x$, this implies $b<x-1$. But now we have an occurrence of 4312 with $x$ as $4, x-1$ as $3, a$ as 1 and $b$ as 2 .

The fact that $x-1$ must appear between $x$ and $n+1$ also shows that the corresponding string has a $B$ before the $T$ corresponding to the value $n+1$; thus $A_{\theta}$ is not a bisecting subset. The case $x=1$ corresponds to the identity permutation; here $C=[n+1]$ and $A_{\theta}=[n]$, which is also non-bisecting.

\footnotetext{
${ }^{3}$ Refer to the first paragraph of Section 2.2.2 for the definition of a bisecting subset.
} 
Finally, we deal with injectivity. It is clear that the wraparound map will associate distinct permutations to distinct (possibly bisecting) subsets; we shall show that if the image $\sigma$ of $A \subseteq[n]$ belongs to a cyclic arrangement $\theta$ of $[n+1]$ and $A \neq A_{\theta}$, then $A$ is a bisecting set. Let $\sigma_{\theta}$ be the image of $A_{\theta}$; we have $\sigma, \sigma_{\theta} \in \theta$, and by construction in both permutations there is an increasing chain starting at position 1 , while the remaining values form a decreasing chain; in $\sigma_{\theta}$, however, the increasing chain is the longest cyclic increasing chain, that is, the longest possible increasing chain (starting at position 1) among all representatives of $\theta$. If $\sigma_{\theta}(1)=x$ and $\sigma(1)=y$, since $\sigma \neq \sigma_{\theta}$ we have that $x<y, y>1$ and the increasing chain of $\sigma$ is strictly contained in that of $\sigma_{\theta}$ and not longest possible. This implies that in $\sigma$ the value $y-1$ is to the right of $n+1$; but then so are all the smaller values, which leaves the increasing chain entirely to the left of the decreasing chain. Thus $A=\{1,2, \ldots, n-y+1\}$, which is a bisecting subset of $[n]$.

3.2 Corollary: The number of (1243)-avoiding cyclic arrangements of $[n]$ is $2^{n-1}-$ $n+1$. They are in a one-to-one correspondence with the Grassmannian permutations of $[n-1]$.

Proof: This follows from (3.1) and the bijective construction of Grassmannian permutations in terms of non-bisecting subsets described in Section 2.2.2.

We remark here that although both the wraparound map and the bijective construction of Grassmannian permutations given in Section 2.2.2 are one-to-one over the set of nonbisecting subsets of $[n]$ but fail to be one-to-one over the entire power set of $[n]$, in the two cases injectivity fails in rather different ways. Indeed, in the case of Grassmannian permutations, all sets of the form $[k]$ for $k \in[0, n]$ (all of which are bisecting sets, except for $[n]$ itself) are mapped to the identity permutation, whereas in the case of the wraparound map, for $k \in[0, n-1]$ the image of $[k]$ is the same as the image of $[n-k, n]$. The wraparound map also has the following interesting link to classical permutation avoidance.

3.3 Proposition: The wraparound map takes the power set of $[n]$ bijectively onto the $\{132,312\}$-avoiding permutations of $[n+1]$.

Proof: We observe that if $A \subseteq[n], \sigma$ is the image of $A$ under the wraparound map and $L, R \subseteq[n+1]$ are respectively the set of positions $i$ such that the label $\sigma(i)$ does not correspond to a $T, B$ (note $L \cup R=[n+1], L \cap R=\{1\}$ ), then:

i $i \in[n]$ is a descent of $\sigma$ if and only if $i \notin A$;

ii for any $i \in L$, there are no values less than $\sigma(i)$ to the left of $i$;

iii for any $i \in R$, there are no values greater than $\sigma(i)$ to the left of $i$.

Considering the entry corresponding to 2 in a potential occurrence of 132 or 312 in $\sigma$, it becomes clear from observations (ii) and (iii) above that $\sigma$ must be $\{132,312\}$-avoiding. 
As we remarked in the proof of 3.1, it is trivial to see that the wraparound map is one-toone. It remains to be proved that any $\{132,312\}$-avoiding permutation $\sigma$ of $[n+1]$ is the image of some subset of $[n]$ under the wraparound map. In view of (i), this subset would have to be the complement in $[n]$ of the set of descents of $\sigma$.

Consider the subset $D \subseteq[2, n+1]$ of integers $i$ such that $i-1$ is a descent of $\sigma$. Now if $j \in D$ then $\sigma\left(j^{\prime}\right)>\sigma(j)$ for all $j^{\prime}<j$ because otherwise we would have $\sigma\left(j^{\prime}\right)<\sigma(j)<\sigma(j-1)$ and since $j^{\prime} \neq j-1,\left\{j^{\prime}<j-1<j\right\}$ would be an occurrence of 132. Similarly (using the fact that $\sigma$ is 312-avoiding) it follows that if $C$ denotes the set $[2, n+1] \backslash D$ and $j \in C$ then for all $j^{\prime}<j$ we have that $\sigma\left(j^{\prime}\right)<\sigma(j)$. This implies that $\sigma$ is decreasing on the set $\{1\} \cup D$ and increasing on the set $\{1\} \cup C$. But then $\sigma$ is the image under the wraparound map of the set $\{i \mid i+1 \in C\}$, which is precisely the complement in $[n]$ of $\{i \mid i+1 \in D\}$, the set of descents of $\sigma$.

Thus, for $n \geq 1$ the number of $\{132,312\}$-avoiding permutations of $[n]$ is $2^{n-1}$. This fact was first shown by Simion and Schmidt [24].

\subsection{The patterns (1324) and (4321)}

Throughout this section, given a permutation $\sigma$ of $[n]$, we shall denote by $\widehat{\sigma}$ the permutation $\sigma \mid n+1$ of $[n+1]$.

3.4 Proposition: A permutation $\sigma$ avoids 132 and 3241 if and only if $\widehat{\sigma}$ avoids (1324).

Proof: Suppose $\sigma$ has an occurrence of 132 or 3241. Clearly any occurrence of 3241 in $\sigma$ is also an occurrence of 3241 , and therefore of (1324), in $\widehat{\sigma}$. If $A$ is an occurrence of 132 in $\sigma$, then $A \cup\{n+1\}$ is an occurrence of 1324 in $\widehat{\sigma}$. Thus in both cases $\widehat{\sigma}$ has an occurrence of (1324).

Conversely, suppose $\widehat{\sigma}$ has an occurrence of $(1324)=\{1324,3241,2413,4132\}$. If $B$ is an occurrence of 1324 in $\widehat{\sigma}$, then $B \backslash\{n+1\}$ contains an occurrence of 132 in $\sigma$. Any occurrences of 3241 in $\widehat{\sigma}$ cannot involve $(n+1)$, so they must also occur in $\sigma$; the same is true for 2413 and 4132, both of which contain an occurrence of 132 .

3.5 Corollary: The (1324)-avoiding cyclic arrangements of $[n]$ are enumerated by the Fibonacci numbers $\mathrm{F}_{2 n-3}$. They are in one-to-one correspondence with non-decreasing Dyck paths of semilength $n-1$.

Proof: Choosing representatives of (1324)-avoiding cyclic arrangements of $[n]$ so that the value $n$ occurs at position $n$, this follows from (3.4), (2.5) and (2.7).

3.6 Proposition: A permutation $\sigma$ avoids 123 and 3412 if and only if $\widehat{\sigma}$ avoids (1234). 
Proof: Suppose $\sigma$ has an occurrence of 123 or 3412 . Clearly any occurrence of 3412 in $\sigma$ is also an occurrence of 3412, and therefore of (1234), in $\widehat{\sigma}$. If $A$ is an occurrence of 123 in $\sigma$, then $A \cup\{n+1\}$ is an occurrence of 1234 in $\widehat{\sigma}$. Thus in both cases $\widehat{\sigma}$ has an occurrence of (1234).

Conversely, suppose $\widehat{\sigma}$ has an occurrence of $(1234)=\{1234,2341,3412,4123\}$. If $B$ is an occurrence of 1234 or 2341 in $\widehat{\sigma}$, then $B \backslash\{n+1\}$ contains an occurrence of 123 in $\sigma$. Any occurrences of 3412 in $\widehat{\sigma}$ cannot involve $(n+1)$, so it must also occur in $\sigma$, and the same is true for 4123, which itself contains an occurrence of 123.

3.7 Corollary: The number of (1234)-avoiding cyclic arrangements of $[n]$ is

$$
2^{n}-\left(\begin{array}{l}
n \\
3
\end{array}\right)-2 n+1
$$

They are in one-to-one correspondence with the simple Dyck paths of semilength $n-1$.

Proof: Choosing representatives of (1234)-avoiding cyclic arrangements of $[n]$ so that the value $n$ occurs at position $n$, applying (3.6) and reversing the patterns, the assertion follows from (2.9) and (2.10).

\section{Orbits}

For a positive integer $n, \mathbb{Z}_{n}$ denotes the usual quotient $\mathbb{Z} / n \mathbb{Z}$. The addition operation on $\mathbb{Z}_{n}$ will be denoted by the usual + , as opposed to $\oplus$, the operation on $[n]$ as defined in Section 3. Whenever it is necessary to specify that + does not stand for the usual addition of integers, or to clarify the value of $n$, we shall use square "lop-sided" brackets with a subscript, e.g. $\lceil 2+5\rfloor_{7}=0,\lceil 2 \oplus 5\rfloor_{7}=7$. Recall that the subset of "invertible" elements of $\mathbb{Z}_{n}$, that is the set $\left\{i \in \mathbb{Z}_{n} \mid \operatorname{gcd}(i, n)=1\right\}$, forms a group with respect to the usual modular multiplication; we shall denote it by $\mathbb{Z}_{n}^{*}$. Also, for integers $p, q$, the notation $p \mid q$ means that $p$ divides $q$.

\subsection{Cyclic Operators and Affine Permutations}

For $a, b \in \mathbb{Z}_{n},\langle a, b\rangle$ denotes the operator from $\mathcal{S}_{n}$ into itself which maps $\sigma$ to $\epsilon_{n}^{-a} \circ \sigma \circ \epsilon_{n}^{b}$ (with $\epsilon_{n}$ as defined in Section 3). The operator $\langle a, b\rangle$ has the effect of rotating the entries to the right by $a$ positions, and rotating their values upward by $b$ positions. Thus for example $\langle 3,1\rangle(21453)=51432$.

Clearly the set $\mathcal{G}_{n}:=\left\{\langle a, b\rangle \mid(a, b) \in \mathbb{Z}_{n}^{2}\right\}$, endowed with functional composition of the operators as a binary operation, has a group structure isomorphic to the direct product of $\mathbb{Z}_{n}$ with itself, i.e. $\left\langle a_{1}, b_{1}\right\rangle \circ\left\langle a_{2}, b_{2}\right\rangle=\left\langle a_{1}+a_{2}, b_{1}+b_{2}\right\rangle$. The equivalence classes of permutations of $[n]$ (under order-equivalence as defined in Section 1 ) obtained when the 
orders on the domain and codomain are both cyclic are precisely the orbits of $\mathcal{S}_{n}$ under the action of $\mathcal{G}_{n}{ }^{4}$. We shall refer to them simply as orbits of length $n$.

A fixed point of an operator $\langle a, b\rangle$ is a permutation $\sigma$ such that $\langle a, b\rangle(\sigma)=\sigma$. An operator $\langle a, b\rangle$ is trivial if $a b=0$, non-trivial otherwise. Clearly the only trivial operator with any fixed points at all is the identity operator $\langle 0,0\rangle$, which fixes all permutations.

For generic positive integers $r, s$, let $\mathcal{F}(r, s)$ denote the set of functions from $[r]$ to $\mathbb{Z}_{s}$.

4.1 Proposition: A non-trivial operator $\langle a, b\rangle \in \mathcal{G}_{n}$ has at least one fixed point if and only if $\operatorname{gcd}(a, n)=\operatorname{gcd}(b, n)$. In this case, let $k=\operatorname{gcd}(a, n)$ and $p=n / k$; the fixed points of $\langle a, b\rangle$ are in one-to-one correspondence with the set $\mathcal{S}_{k} \times \mathcal{F}(k, p)$. Moreover, there exist $\mu, \nu \in \mathbb{Z}_{p}^{*}$ such that $a=\mu k, b=\nu k$ and the fixed points of $\langle a, b\rangle$ are precisely the fixed points of $\langle k, \lambda k\rangle$, where $\lambda=\left\lceil\mu^{-1} \nu\right\rfloor_{p}$.

Proof: Suppose $\langle a, b\rangle$ has a fixed point $\sigma$, and let $k_{1}=\operatorname{gcd}(a, n), k_{2}=\operatorname{gcd}(b, n)$. Then the periods of $a, b$ in $\mathbb{Z}_{n}$ are $p_{1}=n / k_{1}, p_{2}=n / k_{2}$ respectively. Now the operator $\langle a, b\rangle^{p_{1}}=$ $\left\langle a p_{1}, b p_{1}\right\rangle=\left\langle 0, b p_{1}\right\rangle$ fixes $\sigma$, therefore $b p_{1}$ must be 0 , which implies $p_{2} \mid p_{1}$. Similarly we obtain $p_{1} \mid p_{2}$ and we conclude $p_{1}=p_{2}, k_{1}=k_{2}(=k)$. Now by definition of $k_{1}, k_{2}$, we have $a=\mu k, b=\nu k$ for some integers $\mu, \nu$. Since $k=\operatorname{gcd}(a, n)=\operatorname{gcd}(\mu k, n), \mu$ and $n / k=p$ must be coprime, i.e. $\mu \in \mathbb{Z}_{p}^{*}$. Similarly we obtain $\nu \in \mathbb{Z}_{p}^{*}$.

If we set $u=\left\lceil\mu^{-1}\right\rfloor_{p}$ clearly all fixed points of $\langle a, b\rangle$ are also fixed points of $\langle a, b\rangle^{u}=$ $\langle u a, u b\rangle=\left\langle\lceil u \mu\rfloor_{p} k,\lceil u \nu\rfloor_{p} k\right\rangle=\langle k, \lambda k\rangle$ where $\lambda=\left\lceil\mu^{-1} \nu\right\rfloor_{p}$. Also, all fixed points of $\langle k, \lambda k\rangle$ are fixed points of $\langle k, \lambda k\rangle^{\mu}=\left\langle\mu k,\lceil\mu \lambda\rfloor_{p} k\right\rangle=\langle a, b\rangle$.

Since $\sigma$ is an (arbitrary) fixed point of $\langle k, \lambda k\rangle$, for all $i \in[n]$ we must have

$$
\sigma(i \oplus k)=\sigma(i) \oplus \lambda k
$$

and by induction we obtain that for all $s$

$$
\sigma(i \oplus s k)=\sigma(i) \oplus s \lambda k .
$$

Now we partition the domain $[n]$ by considering the cosets in $([n], \oplus)$ with respect to the subgroup generated by $k$, that is, the sets

$$
C_{j}=\{j, j \oplus k, j \oplus 2 k, \ldots, j \oplus(p-1) k\}
$$

for $j \in[k]$. Now as $s$ varies in $\mathbb{Z}_{p}, j \oplus s k$ ranges over $C_{j}$ and since $\lambda$ itself belongs to $\mathbb{Z}_{p}^{*}$, $\lceil s \lambda\rfloor_{p}$ ranges over all of $\mathbb{Z}_{p}$ and $\sigma(j) \oplus s \lambda k$ ranges over the set

$$
\{\sigma(j), \sigma(j)+k, \sigma(j)+2 k, \ldots \sigma(i) \oplus(p-1) k\}=C_{\bar{\jmath}}
$$

where $\bar{\jmath}=\sigma(j) \operatorname{omod}^{5} k$. Thus the image of a coset is a coset, and since $\sigma$ is a permutation of $[n]$, of which the cosets constitute a partition, this is a one-to-one correspondence. Hence

\footnotetext{
${ }^{4}$ For an introduction to the action of groups on sets and the orbit-counting lemma, see for example [7].

${ }^{5}$ Refer to the first paragraph of Section 3 for the definition of the symbol omod.
} 
the map $\bar{\sigma}$ given by $j \mapsto \bar{\jmath}$ is a permutation of $[k]$ satisfying $\sigma\left(C_{j}\right)=C_{\bar{\jmath}}$. At this stage it is clear that $\bar{\jmath}$ depends only on the equivalence class of $\sigma(j)$ modulo $k$, that all bijective assignments of cosets to cosets are achievable, that these assignments are in a one-to-one correspondence with $\mathcal{S}_{k}$ and that the action of $\sigma$ can be broken down into its action on the separate cosets, i.e. in order for a permutation of $[n]$ to be a fixed point of $\langle k, \lambda k\rangle$, it is necessary and sufficient for it to satisfy Equation (8) for all $i \in[k], s \in[p]$.

This equation implies that, for any $j \in[k]$, fixing the value of $\sigma$ on any point in $C_{j}$ uniquely determines $\sigma$ on all of $C_{j}$. Thus, once fixed a coset-to-coset assignment, in order to uniquely determine $\sigma$ we only need to specify which one of the $p$ possible rotations each coset assumes; this we do by specifying, for all $j \in[k]$, the relative position of $\bar{\jmath}$ within $C_{j}$, i.e. the integer $t_{j} \in \mathbb{Z}_{p}$ for which $\sigma\left(j+t_{j} k\right)=\bar{\jmath}$. If we denote by $c_{\sigma}$ the map $j \mapsto t_{j}$, the map $\sigma \mapsto\left(\bar{\sigma}, c_{\sigma}\right)$ is the desired bijection between the set of fixed points of $\langle a, b\rangle$ and $\mathcal{S}_{k} \times \mathcal{F}(k, p)$.

The above proposition shows that the fixed points depend only on $k$ and $\lambda$. This already allows us to easily derive an expression for the total number of orbits of length $n$. This quantity seems to have been first considered by Steggall [27] in 1907, and the result we give here is equivalent to his. We state it mainly for the sake of completeness, and also because to our knowledge this precise formulation does not appear in the literature.

\subsection{Proposition: The total number of orbits of length $n$ is given by}

$$
\frac{1}{n^{2}} \sum_{k p=n} \phi(p)^{2} k ! p^{k}
$$

where $\phi$ is the Euler totient function, i.e. $\phi(i)$ is the number of integers less than $i$ and relatively prime to $i$.

Proof: By the orbit-counting lemma, it is sufficient to take the average over all operators of the number of their fixed points. We consider non-trivial operators first. By Proposition 4.1, for a non-trivial operator $\langle a, b\rangle$ to have any fixed points at all, we must have $\operatorname{gcd}(a, n)=\operatorname{gcd}(b, n)=k$ for some integer $k$ which divides (and is smaller than) $n$, and in this case it must have $k ! p^{k}$ fixed points, where $p k=n$; these are precisely the fixed points of $\langle k, \lambda k\rangle$, where $\lambda \in \mathbb{Z}_{p}^{*}$ is uniquely determined by $a$ and $b$.

For fixed $k$ and $\lambda$, the number of operators which reduce to $k$ is precisely the number of solutions of $\mu \nu^{-1}=\lambda, \mu, \nu=\mathbb{Z}_{p}^{*}$, that is $\left|\mathbb{Z}_{p}^{*}\right|=\phi(p)$. However, there are just as many possibilities for $\lambda \in \mathbb{Z}_{p}^{*}$. As for the trivial operators, the only one with any fixed points at all is the identity operator $\langle 0,0\rangle$, which fixes all $n$ ! of them. The result now follows by observing that $\phi(1)=1$.

For $n$ from 1 to 10, the values of the above formula are: 1, 1, 2, 3, 8, 24, 108, 640, 4492, 36336. More terms are listed in sequence A002619 in the Encyclopedia of Integer Sequences [18]. 
Note that the fixed points of $\langle a, b\rangle$ can be characterized as solutions of the functional equation $\sigma(x \oplus a)=\sigma(x) \oplus b$; we call a solution of this equation for some $a, b$ with $a b \neq 0$ an affine permutation. Proposition (4.1) shows that an equivalent form is

$$
\sigma(x \oplus k)=\sigma(x) \oplus \lambda k
$$

with $k=\operatorname{gcd}(a, n)=\operatorname{gcd}(b, n)$. This characterization makes it easy to see that if $\sigma_{1}, \sigma_{2}$ are fixed points for $\left\langle k, \lambda_{1} k\right\rangle,\left\langle k, \lambda_{2} k\right\rangle$ with $k \mid n$, then $\sigma_{1} \circ \sigma_{2}$ is a fixed point for $\left\langle k, \lambda_{1} \lambda_{2} k\right\rangle$. For $k \mid n$, we denote by $\mathcal{A}_{k}^{(n)}$ the set of solutions of Equation (9) for some $\lambda$. Thus for $k \neq n$, $\mathcal{A}_{k}^{(n)}$ is the set of fixed points in $\mathcal{S}_{n}$ for some operator $\langle a, b\rangle$ with $\operatorname{gcd}(a, n)=\operatorname{gcd}(b, n)=k$, while $\mathcal{A}_{n}^{(n)}$ is just $\mathcal{S}_{n}$, which is also the set of fixed points of the identity operator.

4.3 Proposition: For positive integers $k, p$, consider the group $\mathcal{H}_{k, p}$ with ground set $S_{k} \times \mathcal{F}(k, p) \times \mathbb{Z}_{p}^{*}$ and group operation, denoted by $\odot$, given by

$$
(\alpha, c, \lambda) \odot(\beta, d, \mu)=\left(\alpha \circ \beta, c+\lambda^{-1}(\sigma \circ d), \lambda \mu\right) .
$$

If $n=k p, \mathcal{A}_{k}^{(n)}$ is a subgroup of $S_{n}$ isomorphic to $\mathcal{H}_{k, p}$.

Proof: Note that the assertion is trivial for $k=n$; hence we shall assume $k<n$. Verification that the group structure of $\mathcal{H}_{k, p}$ is well-defined is routine, while the sets of fixed points of $\langle k, \lambda k\rangle$, as $\lambda$ ranges over $\mathbb{Z}_{p}^{*}$, give a partition of $\mathcal{A}_{k}^{(n)}$, so the bijective correspondence between $\mathcal{A}_{k}^{(n)}$ and $\mathcal{H}_{k, p}$ follows from Proposition (4.1). We need to check that composition of permutations in $\mathcal{A}_{k}^{(n)}$ agrees with the group structure of $\mathcal{H}_{k, p}$; the fact that $\mathcal{A}_{k}^{(n)}$ is closed under composition follows easily from Equation (9), but will also emerge from the following proof.

For any permutation $\tau \in \mathcal{A}_{k}^{(n)}$, let $\bar{\tau}, c_{\tau}$ be defined as in the proof of Proposition (4.1). Let $\sigma_{1}, \sigma_{2}$ be arbitrary fixed points of $\left\langle k, \lambda_{1} k\right\rangle,\left\langle k, \lambda_{2} k\right\rangle$ respectively with $\lambda_{1}, \lambda_{2} \in \mathbb{Z}_{p}^{*}$. Then for any $i \in[n]$, writing $i=q k+r$ with $q \in \mathbb{Z}_{p}$ and $r \in[k]$ (notice these are not quite the standard quotient and remainder) we have

$$
\begin{aligned}
\sigma_{1}(q k+r) & =\sigma_{1}\left(r \oplus c_{\sigma_{1}}(r) k \oplus\left\lceil q-c_{\sigma_{1}}(r)\right\rfloor_{p} k\right) \\
& =\sigma_{1}\left(r \oplus c_{\sigma_{1}}(r) k\right) \oplus\left\lceil q-c_{\sigma_{1}}(r)\right\rfloor_{p} \lambda_{1} k \\
& =\bar{\sigma}_{1}(r) \oplus\left\lceil\left(q-c_{\sigma_{1}}(r)\right) \lambda_{1}\right\rfloor_{p} k
\end{aligned}
$$

where (10) is an application of (8) and (11) holds by definition of $\bar{\sigma}_{1}$ and $c_{\sigma_{1}}$. Similarly we obtain

$$
\sigma_{2}(q k+r)=\bar{\sigma}_{2}(r) \oplus\left\lceil\left(q-c_{\sigma_{2}}(r)\right) \lambda_{2}\right\rfloor_{p} k
$$

Now we observe that Equation (11) essentially expresses $\sigma_{1}(q k+r)$ in the form $q^{\prime} k+r^{\prime}$ with $q^{\prime} \in \mathbb{Z}_{p}$ and $r^{\prime} \in[k]$, so substituting this into Equation(12) we derive

$$
\sigma_{2}\left(\sigma_{1}(q k+r)\right)=\bar{\sigma}_{2}\left(\bar{\sigma}_{1}(r)\right)+\left\lceil\left(q-\left(c_{\sigma_{1}}(r)+\lambda_{1}^{-1} c_{\sigma_{2}}\left(\bar{\sigma}_{1}(r)\right)\right)\right)\left(\lambda_{1} \lambda_{2}\right)\right\rfloor_{p} k .
$$

For fixed $i \in[n]$ with $i=q k+r$, we have $i \oplus k=\lceil q+1\rfloor_{p} k \oplus r$, so if we set $\sigma=\sigma_{1} \circ \sigma_{2}$, from Equation (13) we see that for all $i \in[n]$,

$$
\sigma(i+k) \ominus \sigma(i)=\left\lceil\lambda_{1} \lambda_{2}\right\rfloor_{p} k,
$$


i.e. Equation (7) is satisfied with $\lambda=\left\lceil\lambda_{1} \lambda_{2}\right\rfloor_{p}$, which implies that $\sigma$ is a fixed point for $\left\langle k,\left\lceil\lambda_{1} \lambda_{2}\right\rfloor_{p} k\right\rangle$. Moreover, setting $q=0$ in Equation (13) gives, for all $r \in[k]$,

$$
\sigma(r)=\bar{\sigma}_{2}\left(\bar{\sigma}_{1}(r)\right)+\theta_{r} k
$$

for some integer $\theta_{r} \in \mathbb{Z}_{p}$, so that (since $\bar{\sigma}_{2}$ takes values in $\left.[\mathrm{k}]\right) \bar{\sigma}=\bar{\sigma}_{1} \circ \bar{\sigma}_{2}$. Finally, defining the function $c:[k] \rightarrow \mathbb{Z}_{p}$ by $c=c_{\sigma_{1}}+\lambda_{1}^{-1}\left(\bar{\sigma}_{1} \circ c_{\sigma_{2}}\right)$, thanks to Equation (13) the equation

$$
\sigma\left(t_{r} k+r\right)=\bar{\sigma}(r)
$$

reduces to

$$
\left\lceil\left(t_{r}-c(r)\right) \lambda_{1} \lambda_{2}\right\rfloor_{p}=0
$$

and solving for $t_{r}$ gives $c_{\sigma}=c$.

The figure on page 34 gives an illustration of the above isomorphism. Note that, if $a_{1} \mid a_{2}$ and $\left\langle a_{1}, b\right\rangle(\sigma)=\sigma$, then of course $\left\langle a_{2}, t b\right\rangle(\sigma)=\left\langle a_{1}, b\right\rangle^{t}(\sigma)=\sigma$, where $a_{2}=t a_{1}$. So if $k_{1}\left|k_{2}\right| n, \mathcal{A}_{k_{1}}^{(n)}$ is a subgroup of $\mathcal{A}_{k_{2}}^{(n)}$. Thus affine permutations give a subgroup hierarchy of $\mathcal{S}_{n}$ isomorphic to the divisibility lattice of $n$.

Before delving into pattern avoidance, we conclude this section with an easy proposition about generic fixed points that will come in useful in the next section.

4.4 Proposition: A permutation $\sigma$ is a fixed point for the cyclic operator $\langle a, b\rangle$ if and only if $\sigma^{-1}$ is a fixed point for $\langle b, a\rangle$.

Proof: Let $s$ denote the inverse of the fixed point $\sigma$ of $\langle a, b\rangle$. Then $\sigma(s(j) \oplus a)=\sigma\left(s_{j}\right) \oplus b=$ $j \oplus b$. Equivalently, $s(j \oplus b)=s(j) \oplus a$. Since $j$ is arbitrary, $s$ is a fixed point for $\langle b, a\rangle$.

\subsection{Patterns of length up to 4}

For a permutation $\sigma$, we shall indicate by $((\sigma))$ the orbit to which $\sigma$ belongs. Again, as in Section 3, $((\sigma))$ is technically a set of permutations. For patterns of length up to three, the equivalence classes are identical to the cyclic arrangements, so for $t=1,2$ there is only one pattern of length $t$, which can never be avoided as long as $n \geq t$, and the two patterns of length 3 are $((123))$ and $((321))$ which, for $n \geq 3$, can only be avoided by $\left(\left(\rho_{n}\right)\right)$ and $\left(\left(e_{n}\right)\right)$ respectively, where $e_{n}$ denotes the identity permutation of $[n]$. Thus the first interesting case occurs for patterns of length four. Here we have 3 equivalence classes: $((1234)),((4321))$ and $((1243))$. If for some $\sigma \in \mathcal{S}_{n}$, the cyclic arrangement $((\sigma))$ avoids $((1243))$, and it is neither $\left(\left(\rho_{n}\right)\right)$ nor $\left(\left(e_{n}\right)\right)$ then it must must have an occurrence of both $((1234))$ and $((4321))$, and from this it is easy to derive a contradiction. Thus for $n \geq 3$, there are precisely two orbits of length $n$ that avoid $((1243))$, namely $\left(\left(e_{n}\right)\right)$ and $\left(\left(\rho_{n}\right)\right)$. There remain the patterns $((1234))$ and $((4321))$ to be dealt with. These two cases are reverses of each other; we shall consider the pattern $((4321))$.

We remark here that in this context we regain the operation of taking inverses as one of the basic involutions that allow us to reduce one pattern to another; this is because 


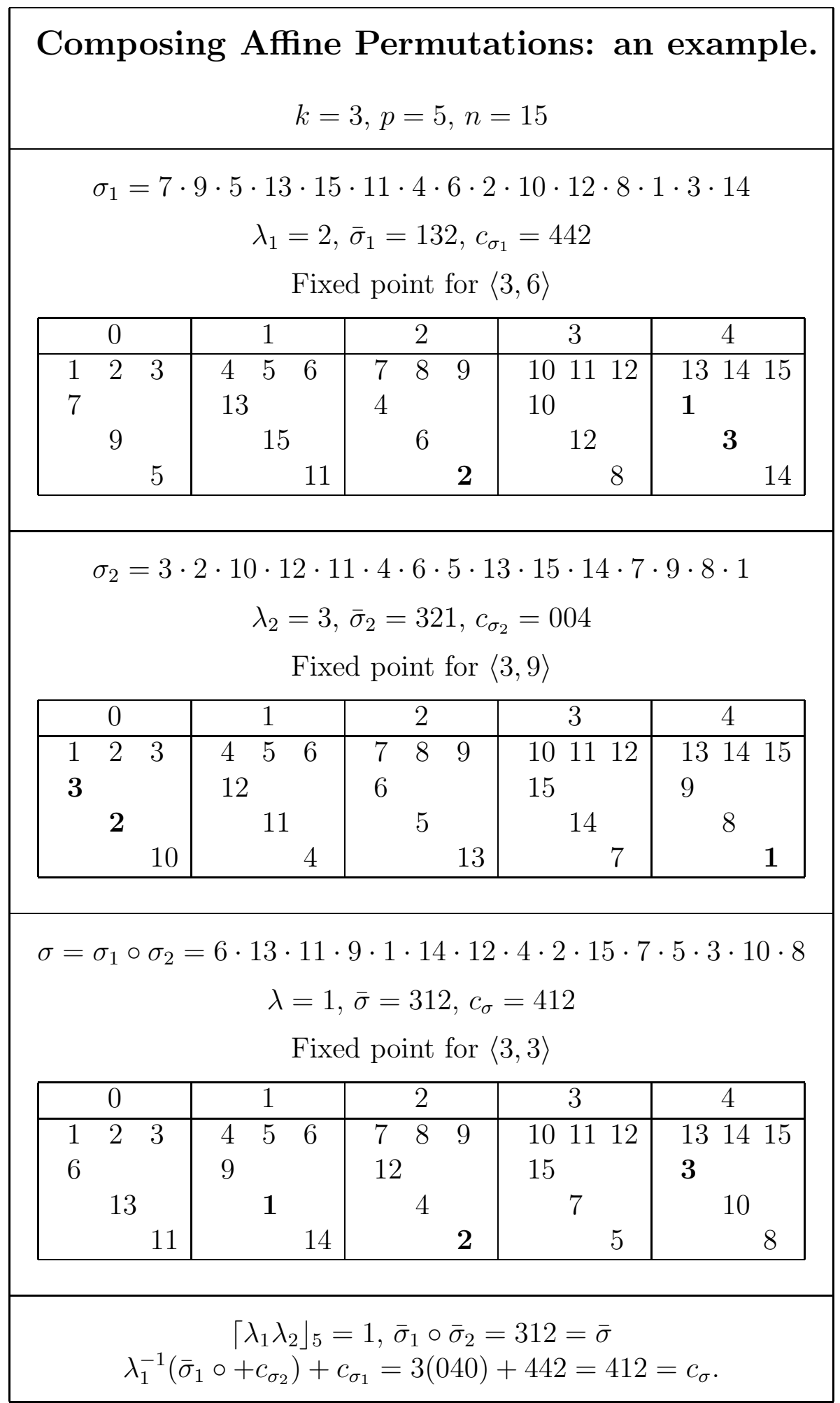


for any equivalence class $\theta$, the set $\left\{\sigma^{-1} \mid \sigma \in \theta\right\}$ is itself an equivalence class. This fact follows easily from the observation that the inverse of $\epsilon_{n}^{i} \circ \sigma \circ \epsilon_{n}^{j}$ is $\epsilon_{n}^{n-j} \circ \sigma^{-1} \circ \epsilon_{n}^{n-i}$, while the observations in Section 3 regarding reversal and complementation still apply to this context.

It turns out that we need to go to patterns of length 6 before taking inverses makes a difference: the 8 patterns of length 5 are reducible to the 3 cases 12345, 12354 and 12534 using reversal and complementation, and we do not get any further merging by allowing inverses as well. However, the 24 patterns of length 6 are reducible to 10 cases using all three operations, and 12 if we were to prohibit inverses. The 10 cases are: $((123456)),((123465)),((123564)),((123654)),((124365)),((124635)),((124653))$, $((125634)),((126453)),((135264))$. Here we shall not be concerned with patterns of length more than four, though.

Since $\epsilon_{n} \circ \rho_{n} \circ \epsilon_{n}=\rho_{n}$, we have that $\left(\rho_{n}\right)=\left(\left(\rho_{n}\right)\right)$; in particular $((4321))=(4321)$, so the ((4321))-avoiding permutations are precisely the (4321)-avoiding permutations studied in Section 3; however, the partition of this set into cyclic arrangements is a refinement of the partition into orbits. Still, using (2.9) and (3.6), we can associate to any ((4321))-avoiding permutation of $[n]$ a simple Dyck path of semilength $n$ by rotating it so as to have the entry $n$ at the end, dropping this entry and taking the corresponding Dyck path (with respect to the sink-or-float bijection). We shall take this to be understood whenever we refer to a ((4321))-avoiding permutation corresponding to a simple Dyck path. Note that going the other way, i.e. from a Dyck path to a ((4321))-avoiding permutation, gives us one representative per cyclic arrangement, whereas we would like one per orbit.

Throughout this section we shall typically be dealing with a permutation $\sigma=\sigma^{\prime} \mid n$ and we shall use heavily the following remark, obtained by pulling together Theorem 2.9, and Propositions 3.6 and 2.8.

4.5 Remark: The following are equivalent:

- $\sigma$ is ((4321))-avoiding;

- $\sigma$ is (4321)-avoiding;

- $\sigma^{\prime}$ is $\{321,2143\}$-avoiding;

- $\sigma^{\prime}$ is the image under the sink-or-float bijection of a simple Dyck path;

- $\sigma^{\prime}$ is Grassmannian or monodipic.

Also when $\sigma$ is a fixed point for some cyclic operator, we shall denote by $\bar{\sigma}$ the first component in the group isomorphism of (4.3), that is $\bar{\sigma}$ as defined in (4.1). Since 4321 is an involution, we also have the following fact which we shall use repeatedly.

4.6 Remark: A permutation $\sigma$ avoids ((4321)) if and only if $\sigma^{-1}$ does.

In order to count the number of orbits that avoid ((4321)), we consider the action of the group $\mathcal{G}_{n}$ on the set of (4321)-avoiding permutations: we count the number of fixed points for each operator and then apply the orbit-counting lemma. 
4.7 Proposition: Suppose $k \mid n$ and the operator $\langle k, \lambda k\rangle \in \mathcal{G}_{n}$ has a ((4321))-avoiding fixed point corresponding to a horizontally simple Dyck path. Then $\lambda \in\{1,2\}$.

Proof: Since $k \neq 0$, clearly $\lambda \neq 0$; suppose by way of contradiction that $\lambda \geq 3$. Let $n=q(\lambda k)+r$ for non-negative integers $q, r$ with $r<\lambda k$. Note that since $\lambda k<n$, $q \geq 1$, and therefore $r<q \lambda k$. Let $\sigma$ be a ((4321))-avoiding fixed point corresponding to a horizontally simple Dyck path; by rotating if necessary, we may assume that the last entry of $\sigma$ is $n$. With this assumption, $\sigma$ is of the form $\sigma^{\prime} \mid n$ for some Grassmannian permutation $\sigma^{\prime}$.

Since $\lambda \geq 3$, we have $n=\lambda(q k)+r \geq 3 q k$; consider the entries at positions $q k, 2 q k, 3 q k$ in $\sigma$. Since the value of the last entry is $n$ and $\sigma$ is a fixed point of $\langle k, \lambda k\rangle$, the first of these three entries has value $q \lambda k$, and the last two are congruent (modulo $n$ ) to $2 q \lambda k$ and $3 q \lambda k$ respectively. Now $q \lambda k \equiv-r$ modulo $n$, and since $r<q \lambda k$, the second of these entries is $q \lambda k-r$. Note that, in particular, thanks to the injectivity of $\sigma$ this implies that $r \neq 0$ (this is also easily deducible from the fact that $\operatorname{gcd}(k, n)=\operatorname{gcd}(\lambda k, n)$ ), and therefore the entries we are considering are all entries of $\sigma^{\prime}$. Thus the entries at positions $q k, 2 q k$ give an inversion in $\sigma^{\prime}$, implying that $\sigma^{\prime}$ has some descent at position $<2 q k$. Since $\sigma^{\prime}$ is Grassmannian, by 2.11 no entry to the right of this descent (in particular, to the right of position $2 q k$ ) can be an excedance.

Suppose now that $2 r<q \lambda k$. Then $q \lambda k-2 r$ would be positive, smaller than $n$ and congruent to $3 q \lambda k$, implying that $\sigma(3 q k)=q \lambda k-2 r$ and that the entries at positions $q k, 2 q k, 3 q k$ give an occurrence of 321 in $\sigma^{\prime}$, a contradiction to the fact that $\sigma^{\prime}$ is the image under the sink-or-float bijection of a Dyck path. If instead $2 r \geq q \lambda k$, then the entry at position $3 q k$ is $2 q \lambda k-r \geq 6 q k-r$ which, since $r<\lambda k \leq 3 k$, is strictly larger than $6 q k-3 k \geq(6 q-3 q) k=3 q k$. Thus, this entry is an excedance, again a contradiction.

4.8 Proposition: If $n=k p$ and $p$ is odd, the number of Grassmannian permutations (horizontally simple Dyck paths) which correspond to ((4321))-avoiding fixed points of $\langle k, 2 k\rangle$ is $2^{k-1}$. If $\sigma$ is such a permutation, then $\bar{\sigma}$ is itself Grassmannian.

Proof: Suppose $\sigma=\sigma^{\prime} \mid n$ is a ((4321))-avoiding fixed point of $\langle k, 2 k\rangle$, with $\sigma^{\prime}$ Grassmannian. Recall from the proof of (4.1) that:

- for all $i \in[k]$, the image of the coset $C_{i}$ is the coset $C_{\bar{\sigma}(i)}$;

- as we move $k$ steps at a time to the right within a given coset in the domain, the value of $\sigma$ goes up by $2 k(\operatorname{omod} n)$;

- given that the image of $C_{i}$ is $C_{j}$, knowing the position of $j$ in $C_{i}$ uniquely determines $\sigma$ on all of $C_{i}$.

Note that since $2 k<n, p$ is at least 3. Also, since $\sigma(n)=n$, we know that the $\sigma(k)=2 k$.

Claim: For all $i \in[k-1]$, at least one of the values $i, i+k$ must occur to the left of position $k$. 
To see this, suppose that both $i$ and $i+k$ lie strictly between positions $k$ and $n$ (note $i<i+k<2 k<n)$ ). If $i$ were to the right of $i+k$, the entries with values $2 k, i+k, i, n$ would give an occurrence of $3214 \in((4321))$ in $\sigma$, so $i$ must be to the left of $i+k$. Now $p$ is odd, so let $p=2 s+1$ for some positive integer $p$. Since $2(s+1)=(2 s+1)+1$ the inverse of 2 modulo $p$ is $s$, so in fact $i+k$ occurs $k(s+1)$ positions to the right of $i$; consider the previous entry in the same coset, i.e. the entry with value $i+2 s k<n, k$ positions to the left of the value $i+k$ and $s k$ positions to the right of the value $i$. Since it is not the last of entry of its coset, its position is at most $n-k=2 s k<2 s k+1$, i.e. this entry gives an excedance. But this excedance is to the right of the value $i$, which is smaller than $\sigma(k)=2 k$ yet in turn occurs to the right of position $k$, implying that there is some descent in $\sigma$ to the left of position $k$, and therefore of the excedance, thus contradicting Proposition (2.11) .

The claim above effectively says that precisely one of $i$ and $i+k$ must occur to the left of position $k$, since each coset has only one position to the left of $k$. Now we know that for all $j \in[k-1], \sigma(j)=\bar{\sigma}(j)$ or $\sigma(j)=\bar{\sigma}(j)+k$. Now suppose that $\sigma$ has a descent to the left of position $k$, i.e. there exists $j<k-1$ such that $\sigma(j)=u, \sigma(j+1)=v$ and $u>v$. Then $u+k$ is larger than, and appears immediately before, $v+k$ (note $u<2 k \Rightarrow u+k<3 k \leq p k=n$ ); none of these entries occupy position $n \in C_{k}$, so we have 2 descents in $\sigma^{\prime}$, contradicting the fact that $\sigma^{\prime}$ is Grassmannian.

Thus the first $k$ entries of $\sigma$ form an increasing subsequence of terms of the form $i$ or $i+k$, with $1 \leq i \leq k$. Let $\widehat{\jmath}$ be the smallest integer for which $\sigma(j)=\bar{\sigma}(j)+k$; then the same must be true for all $j \in[\widehat{\jmath}, k]$, and $\bar{\sigma}$ is increasing over this interval, and over $[\widehat{\jmath}-1]$. So the only possibility for a descent in $\bar{\sigma}$ is position $\widehat{\jmath}-1$; hence, $\bar{\sigma}$ is Grassmannian.

There are $2^{k-1}$ possibilities for choosing one element from each of the sets $\{i, i+k\} i \in$ $[k-1]$ to put to the left of position $k$; from any such choice we can construct a fixed point of $\langle k, 2 k\rangle$ by assigning the cosets in such a way that $\sigma$ is increasing on $[k]$ (placing $2 k$ at position $k$ ). In order to conclude the proof, we need to check that any fixed point $\sigma$ obtained in this way indeed corresponds to a Grassmannian permutation (and therefore is ((4321))-avoiding).

So, suppose we have integers $i_{1}, i_{2}, \ldots, i_{\widehat{\jmath}-1}, i_{\widehat{\jmath}}, \ldots, i_{k}=k$ with $\bar{\sigma}(j)=i_{j}, \widehat{\jmath}$ defined as above, and for $a \in[n]$

$$
\sigma(a)=f(a) \operatorname{omod} p
$$

where

$$
f(j+z k)= \begin{cases}i_{j}+2 z k & \text { if } 1 \leq j \leq \widehat{\jmath}-1 \\ i_{j}+(2 z+1) k & \text { if } \widehat{\jmath} \leq j \leq k\end{cases}
$$

for $z \in[0, p-1]$, and suppose further that $\sigma$ is increasing on $[k]$ (by construction). Then $f$ is increasing on all intervals $[1+z k, k+z k]$ with $z \in[0, p-1]$, but also since $i_{k}=k$, we have for all $z \in[0, p-2]$ that $f((1+z) k)<f((1+z) k+1)$, so $f$ is in fact increasing on all of $[n]$. Thus $\sigma$ will satisfy $\sigma(a)<\sigma(a+1)$ except possibly for those $a \in[n-1]$ for which there exists a positive integer $\theta$ such that $f(a) \leq \theta n<f(a+1)$. We claim that the only such value of $a$ is $\widehat{\jmath}-1+s k$ (recall $p=2 s+1$ ). In fact 


$$
\begin{aligned}
f(\widehat{\jmath}-1+s k) & =(\widehat{\jmath}-1)+2 s k \\
& <(2 s+1) k=p k \\
& =n=p k \\
& <p k+\widehat{\jmath}=(2 s+1)+\widehat{\jmath} \\
& =f(\widehat{\jmath}+s k)=n+\widehat{\jmath} \\
& <2 n=2 p k=k+(2(p-1)+1) k \\
& =f(n) .
\end{aligned}
$$

Thus $\sigma$ is Grassmannian, and therefore truncating the last entry gives a Grassmannian permutation (horizontally simple Dyck path) corresponding to a ((4321))-avoiding permutation.

4.9 Proposition: If $n=k p, \lambda \in \mathbb{Z}_{p}^{*}$ and the operator $\langle k, \lambda k\rangle$ has at least one ((4321))avoiding fixed point, then precisely one of the following holds:

$I \lambda=1$ and all the ((4321))-avoiding fixed points of $\langle k, \lambda k\rangle$ correspond to the identity permutation;

II $\lambda=2$ and all the ((4321))-avoiding fixed points of $\langle k, \lambda k\rangle$ correspond to Grassmannian, but not monodipic, permutations (horizontally simple, but not vertically simple, Dyck paths);

III $\lambda=\left\lceil 2^{-1}\right\rfloor_{p}$ and all the ((4321))-avoiding fixed points of $\langle k, \lambda k\rangle$ correspond to monodipic, but not Grassmannian, permutations (vertically simple, but not horizontally simple, Dyck paths);

$$
\text { IV } p=3 \text { and } \lambda=2=\left\lceil 2^{-1}\right\rfloor_{p} .
$$

Proof: Let $\sigma=\sigma^{\prime} \mid n$ be a $((4321))$-avoiding fixed point of $\langle k, k\rangle$. Then $\sigma^{\prime}$ must be Grassmannian or monodipic. Suppose that $\lambda=1$, and that $\sigma^{\prime}$ is Grassmannian (and therefore $\sigma$ is itself Grassmannian). Since $\sigma$ is a fixed point for $\langle k, k\rangle$, for any $j \in[k]$ the values $\bar{\sigma}(j), \bar{\sigma}(j)+k, \bar{\sigma}(j)+2 k, \ldots \bar{\sigma}(j)+(p-1) k$ occur in this cyclic order and together occupy the positions $j, j+k, j+2 k, \ldots j+(p-1) k$; suppose that they do not start at the beginning, i.e. that $\sigma(i) \neq \bar{\sigma}(i)$. Then $\bar{\sigma}(i)+(p-1) k$ occurs to the left of $\bar{\sigma}(i)$ and therefore there must be a descent somewhere in $\sigma$ to the left of the value $\bar{\sigma}(i)$, yet this entry gives an excedance, contradicting Proposition (2.11). Thus for all $j \in[k]$, $\bar{\sigma}(j)=\sigma(j)$. Now any descent in $[k]$ would be repeated in every interval $[z k+1,(z+1) k]$, $z \in[p-1]$, and this would give at least two descents in $\sigma$ (note $k<n \Rightarrow p \geq 2$ ). So $\sigma$ is increasing on $[k]$ and this shows it must be the identity. If instead $\sigma^{\prime}$ is monodipic, then $\left(\sigma^{\prime}\right)^{-1}$ is Grassmannian, and by (4.4) and (4.6) $\sigma^{-1}=\left(\sigma^{\prime}\right)^{-1} \mid n$ is a (4321)-avoiding (equivalently, ((4321))-avoiding) fixed point of $\langle k, k\rangle$, so the previous argument applies to $\sigma^{-1}$, implying that $\sigma$ is the identity permutation. 
Now we assume $\lambda \neq 1$. Suppose $\sigma_{1}^{\prime}$ is Grassmannian, $\sigma_{2}^{\prime}$ is monodipic, and both $\sigma_{1}^{\prime} \mid n$ and $\sigma_{2}=\sigma_{2}^{\prime} \mid n$ are ((4321))-avoiding fixed points of $\langle k, \lambda k\rangle$ (corresponding to a horizontally and vertically simple Dyck path respectively). Since $\lambda \neq 1$, by (4.7) $\lambda=2$, and since $\sigma_{2}^{\prime} \mid n$ is monodipic, $\left(\sigma_{2}^{\prime}\right)^{-1}$ is Grassmannian. Moreover, by $(4.4), \sigma_{2}^{-1}=\left(\sigma_{2}^{\prime}\right)^{-1} \mid n$ is a fixed point for $\langle\lambda k, k\rangle$ or equivalently (by 4.1) for $\left\langle k,\left\lceil\lambda^{-1}\right\rfloor_{p} k\right\rangle$. Since $\sigma_{2}$ is ((4321))-avoiding, by (4.6) so is $\sigma_{2}^{-1}$, so again by (4.7) $\left\lceil\lambda^{-1}\right\rfloor_{p}=2$. Thus $\lambda=\left\lceil\lambda^{-1}\right\rfloor_{p}=2$, i.e. $p=3$. So if $\lambda \neq 1$ and $p \neq 3$, the operator $\langle k, \lambda k\rangle$ cannot have ((4321))-avoiding fixed points that correspond to vertically simple Dyck paths, and simultaneously fixed points (not even the same ones) that correspond to horizontally simple Dyck paths; moreover, the existence of fixed points of the latter type implies $\lambda=2$, and of the former type $\left[\lambda^{-1}\right\rfloor_{p}=2$. But they must all correspond to vertically or horizontally simple Dyck paths, so precisely one of (II) and (III) must hold.

4.10 Proposition: If $n=3 k$, the Grassmannian monodipic permutations (vertically and horizontally simple Dyck paths) that correspond to ((4321))-avoiding fixed points of $\langle k, 2 k\rangle$ are precisely the ones of the form

$$
1 \cdot 2 \cdots x \cdot y \cdot y+1 \cdots y+k-1 \cdot x+1 \cdot x+2 \cdots y-1 \cdot y+k \cdot y+k+1 \cdots n
$$

for $0 \leq x \leq k-1$ and $y=x+k+1$.

Proof: Let $\sigma=\sigma^{\prime} \mid n$ be a ((4321))-avoiding fixed point of $\langle k, 2 k\rangle$ with $\sigma^{\prime}$ Grassmannian and monodipic. Then $\sigma$ is itself Grassmannian and monodipic. We shall think of $\sigma$ in terms of the corresponding escalating Dyck path (via the sink-or-float bijection). Since the identity permutation is not a fixed point of $\langle k, 2 k\rangle$, this path has precisely one long vertical edge and one long horizontal edge, clearly of the same length, with some hilltops before the long vertical edge and after the long horizontal edge, and some peaks in between the two long edges, all at the same height.

Let $t$ be the position of the first floating step after the long vertical edge (the first excedance, and the only dip) and $u$ the position of the floating step of the long horizontal edge (the last excedance, and the only descent). Now $\sigma(n)=n$, so $\sigma(k)=2 k$; hence $k$ is an excedance, $t \leq k \leq u$, the vertical edge has a length of $k+1$ and $\sigma(j)$ exceeds $j$ by $k$ for all $j: t \leq j u$. In particular $\sigma(t)=t+k$, so $\sigma(t+k)=\sigma(t) \oplus 2 k=t \oplus 3 k=t$. Also, all points before position $t$ are fixed points of $\sigma$, so if $t \geq 2, \sigma(t+k-1)=\sigma(t-1)+2 k=t+2 k-1$, while if $t=1 \sigma(t+k-1)=2 k$. In both cases $\sigma(t+k-1)>t+k=\sigma(t)$, so $t+k-1$ is a descent. But then $u=t+k-1$ and, fixed $t$, this completely determines $\sigma$ as a (possibly empty) sequence of $t-1$ fixed points, followed by $u-t+1=k$ points on which $\sigma$ exceeds its argument by $k$, then an increasing sequence of $k$ deficiencies, and finally $n-2 k-t+1$ more fixed points. That such a permutation is ((4321))-avoiding is just a consequence of the fact that it corresponds to a simple Dyck path, and to see that it must be a fixed point for $\langle k, 2 k\rangle$ we observe that the only places where $\sigma(a \oplus 1)=\sigma(a) \oplus 1$ fails is at the points $a_{1}=t \ominus 1, a_{2}=t \oplus k \ominus 1$, which satisfy $a_{1} \oplus k=a_{2}, \sigma\left(a_{2}\right)=\sigma\left(a_{1}\right) \oplus 2 k$ (recall $n=3 k)$. 
4.11 Corollary: If $n=3 k$, the number of simple Dyck paths corresponding to ((4321))avoiding fixed points of $\langle k, 2 k\rangle$ is $2^{k}-k$.

Proof: By (4.8), there are precisely $2^{k-1}$ horizontally simple Dyck paths of this kind. As for the vertically simple ones, taking inverses gives a bijection between the ((4321))avoiding fixed points of $\langle k, 2 k\rangle$ corresponding to vertically simple Dyck paths and the ((4321))-avoiding fixed points of $\langle 2 k, k\rangle$ corresponding to horizontally simple Dyck paths. But by (4.1), the fixed points of $\langle 2 k, k\rangle$ are the fixed points of $\left\langle k,\left\lceil 2^{-1}\right\rfloor_{3} k\right\rangle=\langle k, 2 k\rangle$. So again by (4.8) there are $2^{k-1}$ vertically simple Dyck paths corresponding to ((4321))avoiding fixed points of $\langle k, 2 k\rangle$. By (4.10) precisely $k$ of these are vertically and horizontally simple. Thus the total number is $2.2^{k-1}-k$.

4.12 Theorem: The number of ((4321))-avoiding orbits of $[n]$ is given by

$$
\frac{1}{n}\left(2^{n}-\left(\begin{array}{l}
n \\
3
\end{array}\right)-n-\frac{\widehat{\frac{2 n}{3}}}{\underline{3}}+\sum_{\substack{2 \nmid p \mid n \\
1<p \leq n}} \phi(p) 2^{\frac{n}{p}}\right)
$$

where $\phi$ is the Euler totient function and $\underline{\widehat{x}}$ denotes $x$ if $x$ is an integer, and 0 otherwise.

Proof: By the orbit-counting lemma, it is sufficient to take the average over all $\langle a, b\rangle \in \mathcal{G}_{n}$ of the number of ((4321))-avoiding fixed points of $\langle a, b\rangle$. The identity operator fixes all the ((4321))-avoiding permutations, of which there are $n$ times the number of $((4321))$ avoiding cyclic arrangements, i.e. $n\left(2^{n}-\left(\begin{array}{l}n \\ 3\end{array}\right)-2 n+1\right)$ (using 3.7). Clearly, no other trivial operator has any fixed points at all.

For any non-trivial operator with at least one ((4321))-avoiding fixed points $\langle a, b\rangle$, by (4.1) the set of fixed points is precisely the set of fixed points of $\langle k, \lambda k\rangle$ where $k=$ $\operatorname{gcd}(a, n)=\operatorname{gcd}(b, n), a=\mu k, b=\nu k, \lambda=\left\lceil\mu^{-1} \nu\right\rfloor_{p}$ and $n=k p$. We need to calculate how many operators reduce to each one of the four scenarios in (4.9). Clearly $\lambda=1 \Leftrightarrow \mu=\nu$, so a non-trivial operator will reduce to situation (I) if and only if it is of the form $\langle a, a\rangle$. There are $n-1$ of these operators, each of which has as ((4321))-avoiding fixed points all the $n$ rotations of the identity permutation. That takes care of case (I) and we may now assume $2 \nmid p$.

To deal with cases (II) and (III), we observe that the equations $\lambda=2, \lambda=\left\lceil 2^{-1}\right\rfloor_{p}$ are equivalent to $\mu=2 \nu$ and $\nu=2 \mu\left(\nu, \mu \in \mathbb{Z}_{p}^{*}\right)$ respectively, so they each have $\left|\mathbb{Z}_{p}^{*}\right|=\phi(p)$ solutions. In case (II), by (4.8) there are $2^{k-1}$ horizontally simple Dyck paths corresponding to ((4321))-avoiding fixed points, and by (4.9) itself, these are the only ones. In case (III), taking inverses gives a bijection between the ((4321))-avoiding fixed points of $\left\langle k,\left\lceil 2^{-1}\right\rfloor_{p}\right\rangle k$ and those of $\langle k, 2 k\rangle$ (using once more (4.6), (4.4) and (4.1)), so again the total number is $2^{k-1}$. Thus, for each $p \mid n, p \neq 3$, there are $\phi(p)$ operators that reduce to situation (II) and just as many that reduce to situation (III), each of which has $2^{k-1} n((4321))$-avoiding fixed points ( $n$ per cyclic arrangement). 
As for case (IV), the equation $\lambda=2$ with $p=3$ has the two solutions $\mu=1, \nu=2$ and $\mu=2, \nu=1$. Thus, if $3 \mid n$, there are another two operators, namely $\langle\bar{k}, 2 \bar{k}\rangle$ and $\langle 2 \bar{k}, \bar{k}\rangle$ where $3 \bar{k}=n$, that have the same (non-empty) set of (4321)-avoiding fixed points. By (4.11), there are precisely $n\left(2^{\bar{k}}-\bar{k}\right)$ of these.

Using the above considerations to take the sum over all operators of the number of their fixed points, dividing by $n^{2}$ (the total number of operators) and simplifying gives the desired formula.

The first 20 values of the sequence given by the above formula are: 1, 1, 2, 2, 5, 7, 14, 24, 49, 91, 172, 324, 609, 1145, 2160, 4060, 7671, 14525, 27544, 52374.

\section{Acknowledgements}

My sincere gratitude goes to Einar Steingrímsson for his initiative, guidance, generosity and enthusiastic support, without which this work would not have been possible. In particular, the presentation has benefited from his careful reading and numerous helpful suggestions. Further improvements in presentation, simplification of some proofs and several pointers to the literature are due to an anonymous referee, who also made the crucial observation which led to Proposition 3.3. I also wish to thank the RISC combinatorics group at the University of Linz, in particular Peter Paule, for allowing me to use their software.

\section{References}

[1] R.M. Adin and Y. Roichman. Equidistribution and sign-balance on 321-avoiding permutations. Available at arXiv:math.C0/03044297.

[2] N. Alon and J.H. Spencer. The Probabilistic Method. John Wiley \& Sons, Inc., New York, 1992.

[3] E. Barcucci, A. Del Lungo, S. Fezzi, and R. Pinzani. Nondecreasing Dyck paths and q-Fibonacci numbers. Discrete Math., 170:211-217, 1997.

[4] E. Barcucci, R. Pinzani, and R. Sprugnoli. Directed column-convex polyominoes by recurrence relations. In TAPSOFT '93: theory and practice of software development (Orsay, 1993), volume 668 of Lecture Notes in Comput. Sci., pages 282-298. Springer, Berlin, 1993.

[5] S. Billey, W. Jockusch, and R.P. Stanley. Some combinatorial properties of Schubert polynomials. J. Algebraic Combin., 2(4):345-374, 1993.

[6] P. Bränden, A. Claesson, and E. Steingrímsson. Catalan continued fractions and increasing subsequences in permutations. Discrete Math., 258:275-287, 2002. 
[7] P.J. Cameron. Permutation groups. In Handbook of Combinatorics, pages 611-645. Elsevier Science B.V., Amsterdam, 1995.

[8] P.J. Cameron. Homogeneous permutations. Electron. J. Combin., 9(2):\#R2, 2002.

[9] E. Deutsch. A bijection on Dyck paths and its consequences. Discrete Math., 179(13):253-256, 1998.

[10] E. Deutsch. Dyck path enumeration. Discrete Math., 204:167-202, 1999.

[11] E. Deutsch and H. Prodinger. A bijection between directed column-convex polyominoes and ordered trees of height at most three. Theoret. Comput. Sci., 307:319-325.

[12] S. Elizalde. Fixed points and excedances in restricted permutations. Available at arXiv:math.CO/0212221, 2002.

[13] K. Eriksson and S. Linusson. Combinatorics of Fulton's essential set. Duke Math. J., 85(1):61-76, 1996.

[14] H. Kok. Connected Orderable Spaces. Mathematisch Centrum, Amsterdam, 1973.

[15] C. Krattenthaler. Permutations with restricted patterns and Dyck paths. Adv. Appl. Math., 27:510-530, 2001.

[16] C. Krattenthaler. Private communication. 2003.

[17] A. Lascoux and M.-P. Schützenberger. Schubert polynomials and the LittlewoodRichardson rule. Lett. Math. Phys., 10(2-3):111-124, 1985.

[18] N. J. A. Sloane, editor (2003). The on-line encyclopedia of integer sequences, published electronically at http://www.research.att.com:80/ njas/sequences/.

[19] T.V. Narayana. Sur les treillis formés par les partitions d'un entier et leurs applications à la théorie des probabilités. C. R. Acad. Sci. Paris, 240:1188-1189, 1955.

[20] M. Petkov̌sek, H. Wilf, and D. Zeilberger. $A=B$. A. K. Peters, Wellesley, MA 1996.

[21] A. Reifegerste. The excedances and descents of bi-increasing permutations. Available at arXiv:math.CO/0212247, 2002.

[22] A. Reifegerste. Refined sign-balance on 321-avoiding permutations. Available at arXiv:math.CO/0305327, 2003.

[23] A. Robertson, D. Saracino, and D. Zeilberger. Refined restricted permutations. Available at arXiv:math. CO/0203033.

[24] R. Simion and F.W. Schmidt. Restricted permutations. Europ. J. Combinatorics, 6:383-406, 1985. 
[25] L.J. Slater. Generalized Hypergeometric Functions. Cambridge University Press, London, 1966.

[26] R.P. Stanley. Catalan addendum (2003). Available at http://www-math.mit.edu/〜rstan/ec/.

[27] J.E.A. Steggall. On the numbers of patterns which can be derived from certain elements. Messenger Math., 37:56-61, 1907.

[28] E. Weisstein, editor (2003). Pascal's triangle. In Eric Weisstein's World of Mathematics. Available at http://mathworld.wolfram.com/PascalsTriangle.html.

[29] J. West. Generating trees and forbidden subsequences. Discrete Math., 157(1-3):363$374,1996$. 